\title{
MDM2 inhibition in combination with endocrine therapy and CDK4/6 inhibition for the treatment of ER-positive breast cancer
}

Neil Portman ${ }^{1,2+}$ (D) Heloisa H. Milioli ${ }^{1,2+}$, Sarah Alexandrou ${ }^{1,2}$, Rhiannon Coulson ${ }^{1,3,4}$, Aliza Yong ${ }^{1}$, Kristine J. Fernandez ${ }^{1}$, Kee Ming Chia', Ensar Halilovic ${ }^{5}$, Davendra Segara ${ }^{1,2}$, Andrew Parker ${ }^{1,2}$, Sue Haupt ${ }^{3,4}$, Ygal Haupt ${ }^{3,4}$, Wayne D. Tilley ${ }^{6}$, Alex Swarbrick ${ }^{1,2}$, C. Elizabeth Caldon ${ }^{1,2 \dagger}$ and Elgene Lim ${ }^{1,2^{*}}$

\section{Abstract}

Background: Resistance to endocrine therapy is a major clinical challenge in the management of oestrogen receptor (ER)-positive breast cancer. In this setting, p53 is frequently wildtype and its activity may be suppressed via upregulation of its key regulator MDM2. This underlies our rationale to evaluate MDM2 inhibition as a therapeutic strategy in treatment-resistant ER-positive breast cancer.

Methods: We used the MDM2 inhibitor NVP-CGM097 to treat in vitro and in vivo models alone and in combination with fulvestrant or palbociclib. We perform cell viability, cell cycle, apoptosis and senescence assays to evaluate antitumour effects in p53 wildtype and p53 mutant ER-positive cell lines (MCF-7, ZR75-1, T-47D) and MCF-7 lines resistant to endocrine therapy and to CDK4/6 inhibition. We further assess the drug effects in patient-derived xenograft (PDX) models of endocrine-sensitive and endocrine-resistant ER-positive breast cancer.

Results: We demonstrate that MDM2 inhibition results in cell cycle arrest and increased apoptosis in p53-wildtype in vitro and in vivo breast cancer models, leading to potent anti-tumour activity. We find that endocrine therapy or CDK4/ 6 inhibition synergises with MDM2 inhibition but does not further enhance apoptosis. Instead, combination treatments result in profound regulation of cell cycle-related transcriptional programmes, with synergy achieved through increased antagonism of cell cycle progression. Combination therapy pushes cell lines resistant to fulvestrant or palbociclib to become senescent and significantly reduces tumour growth in a fulvestrant-resistant patient-derived xenograft model.

Conclusions: We conclude that MDM2 inhibitors in combination with ER degraders or CDK4/6 inhibitors represent a rational strategy for treating advanced, endocrine-resistant ER-positive breast cancer, operating through synergistic activation of cell cycle co-regulatory programmes.

Keywords: Breast cancer, MDM2, Oestrogen receptor, CDK4/6 inhibitor, p53

\footnotetext{
* Correspondence: e.lim@garvan.org.au

${ }^{\dagger}$ Neil Portman, Heloisa H. Milioli, C. Elizabeth Caldon and Elgene Lim contributed equally to this work.

'Garvan Institute of Medical Research, Darlinghurst, Sydney, NSW 2010,

Australia

${ }^{2}$ St. Vincent's Clinical School, Faculty of Medicine, University of New South Wales Sydney, Sydney, NSW 2010, Australia

Full list of author information is available at the end of the article
}

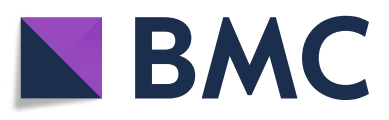

(c) The Author(s). 2020 Open Access This article is licensed under a Creative Commons Attribution 4.0 International License, which permits use, sharing, adaptation, distribution and reproduction in any medium or format, as long as you give appropriate credit to the original author(s) and the source, provide a link to the Creative Commons licence, and indicate if changes were made. The images or other third party material in this article are included in the article's Creative Commons licence, unless indicated otherwise in a credit line to the material. If material is not included in the article's Creative Commons licence and your intended use is not permitted by statutory regulation or exceeds the permitted use, you will need to obtain permission directly from the copyright holder. To view a copy of this licence, visit http://creativecommons.org/licenses/by/4.0/ The Creative Commons Public Domain Dedication waiver (http://creativecommons.org/publicdomain/zero/1.0/) applies to the data made available in this article, unless otherwise stated in a credit line to the data. 


\section{Background}

Oestrogen receptor (ER)-positive breast cancer accounts for $\sim 70 \%$ of breast cancer diagnoses. For many patients, the disease is treated effectively by surgery and adjuvant endocrine therapy. Unfortunately, $\sim 35 \%$ of patients receiving endocrine therapy relapse with resistant disease, either through inherent resistance to treatment or the emergence of acquired endocrine resistance $[1,2]$. Resistant disease that retains ER positivity is typically managed by treating with additional lines of endocrine therapy. Recently, the addition of cyclin-dependent kinase 4 and 6 (CDK4/6) inhibitors to endocrine therapy has become the new standard of care for first- and second-line treatment for advanced breast cancer [3]. However, even with the addition of CDK4/6 inhibitors, resistance develops on average within 2-3 years and the need to identify novel targets and therapies in the resistant setting remains pressing $[3,4]$.

When functioning normally, p53 is activated in response to diverse genotoxic stimuli and initiates transcriptional programmes that lead to cell cycle inhibition, DNA repair and, in the case of irreversible damage, apoptosis. In ER-positive breast cancer and liposarcoma, the incidence of p53 mutation is relatively low [5] but there is an increased incidence of dysregulation of the major p53 regulatory proteins, MDM2 and MDM4 [6]. MDM2 and MDM4 interact directly with p53 to inhibit its transcriptional activity and promote its relocation to the cytoplasm. MDM2 additionally ubiquitinates p53 to initiate its proteosomal degradation [7-10]. Consequently, the pharmacological reactivation of $\mathrm{p} 53$ function through the inhibition of MDM2 has attracted significant attention as a potential therapeutic strategy [5].

In breast cancer, although the incidence of amplification or mutation of MDM2 is relatively low, increased abundance of MDM2 protein occurs in 38\% of all breast cancers and is more frequent among ERpositive than in ER-negative tumours $[6,11]$. There is significant interaction between the MDM2/p53 axis and ER signalling. $M D M 2$ is a transcriptional target of ER, and MDM2 protein interacts directly with ER $[12,13]$. ER also regulates and interacts with p53 [14, 15] and activation of ER by either its cognate ligand or by selective ER modulators such as tamoxifen inhibits the activity of p53 [14]. Simultaneous inhibition of the MDM2/p53 interaction using small molecule inhibitors and degradation of ER via the selective oestrogen receptor degrader fulvestrant can synergistically reduce proliferation of cell line models and xenografts $[14,16]$. Curiously, this synergy occurs without the significant induction of apoptosis [16]. An unresolved question is how MDM2 inhibition synergises with endocrine therapy, and whether outcomes would be improved in combination with the new standard-of-care treatment, CDK4/6 inhibitors.

In this study, we characterised the anti-tumour effect of p53 activation via MDM2 inhibition using the small molecule inhibitor NVP-CGM097-a dihydroisoquinolinone derivative currently being evaluated in a phase I clinical trial $[17,18]$-in endocrine-resistant and endocrine-sensitive in vitro and in vivo models of ERpositive breast cancer. We show synergistic tumour cell inhibition in vitro in combination with either fulvestrant or palbociclib specifically via cell cycle arrest pathways, rather than by a general upregulation of p53 activity that includes apoptosis. We then demonstrate that in endocrine- and CDK4/6 inhibitor-resistant in vitro models, MDM2 inhibition is potentiated by combination with endocrine therapy or CDK4/6 inhibition and that this occurs via an increase in senescence compared to MDM2 inhibition alone.

\section{Methods}

\section{Cell culture and reagents}

MCF7 cells (Michigan Cancer Foundation), T-47D and ZR75-1 cells (American Type Culture Collection) lines were verified through short tandem repeat profiling and tested negative for mycoplasma contamination at the commencement of the project. Frozen stocks of each line used were generated at the commencement of the project and defrosted cells were cultured for between 10 and 20 passages. Parental cell lines were cultured in RPMI 1640 media (Thermo Fisher) supplemented with $10 \%$ foetal bovine serum (GE Healthcare) and $20 \mathrm{mM}$ HEPES (Thermo Fisher). Fulvestrant-resistant (FasR) and palbociclib-resistant (PalbR) MCF-7 derivatives were developed and cultured in phenol red-free RPMI 1640 (Thermo Fisher) supplemented with $5 \%$ charcoal stripped foetal bovine serum (GE Healthcare), $20 \mathrm{mM}$ HEPES and $10 \mathrm{pM}$ $17 \beta$-estradiol (Sigma). Resistance to fulvestrant was developed by the continuous addition of $100 \mathrm{nM}$ fulvestrant (Assay Matrix) over 12 months until the cells proliferated with a constant doubling time, and cells were subsequently maintained with $100 \mathrm{nM}$ fulvestrant. Resistance to palbociclib was developed by the addition of 500 $\mathrm{nM}$ palbociclib (Selleckchem) for 8 months until the cells proliferated with a constant doubling time, and cells were subsequently maintained with $500 \mathrm{nM}$ palbociclib. For experiments that required a no-drug treatment arm, selection was removed for $48 \mathrm{~h}$ prior to commencement of treatment. Stock solutions of each drug $(10,000 \times)$ were prepared in DMSO (NVP-CGM097, fulvestrant), ethanol (17 $\beta$-estradiol) or water (palbociclib). All cells were cultured under $5 \% \mathrm{CO}_{2}$ in a humidified incubator at $37^{\circ} \mathrm{C}$. 


\section{Western blot analysis}

All Western blot analyses were repeated on at least three separate occasions using independently generated lysates. Cells were lysed in RIPA buffer supplemented with Halt Protease and Phosphatase Inhibitor Cocktail (Thermo Fisher). Protein lysates were separated by electrophoresis over 4-15\% mini-Protean TGX gels (BioRad) and transferred to $0.45 \mu \mathrm{m}$ Immobilon-FL polyvinylidene fluoride membrane (Millipore). Proteins were detected by immunoblotting with specific primary antibodies and subsequent detection by fluorescent conjugated secondary antibodies [IRDye 680RD Donkey anti-mouse (\#926-68072, LI-COR) or IRDye $800 \mathrm{CW}$ Donkey anti-Rabbit (\#926-32213, LI-COR)]. Fluorescent signals were visualised using the Odyssey CLx Imaging System (LI-COR). Anti-p21 12D1 (2947) and anti-p53 (9282) were sourced from Cell Signalling, and antiMDM2 2A10 (ab16895) was sourced from Abcam.

\section{Proliferation assays}

Cells were seeded into 96-well plates at a density of 3000 cells in $100 \mu \mathrm{l}$ media per well. Treatment was delivered via replacement of the media in each well with fresh media containing the indicated concentrations of drugs the following day. Mitochondrial activity (as an indication of cell viability) was assessed using the alamar blue assay (Thermo Fisher) according to the manufacturer's instructions 2 days after treatment. Experiments were performed in three independent replicates. Growth inhibition was calculated using the formula 1 - $\left(\frac{\text { treated }}{\text { vehicle }}\right)$. Alternatively, proliferation was measured by cell number using the IncucyteTM live-cell imaging system (Essen Bioscience). Cells were plated at 1000-3000 cells per well in a 96 well plate in 40 wells/cell line, and cell number determined by imaging every $1-2$ days for 2 weeks. IC50 values were calculated from non-linear regression analyses performed in GraphPad Prism (version 7.04, GraphPad Software). Synergy was quantified using a dose-effect approach with a constant dose ratio design as per Chou and Talalay [19]. Combination indices (C.I.) were calculated using CompuSyn software (ComboSyn, Inc) such that a $\log _{2}$ (C.I. $)<0$ indicates synergy.

\section{Senescence assay}

Senescence was assayed by visualising $\beta$-galactosidase activity using Senescence $\beta$-galactosidase staining kit (Cell Signalling) according to the manufacturer's instructions. Three fields of approximately 100 cells were scored for each of three independent replicates for each treatment condition.

\section{Flow cytometry}

Flow cytometry was performed on a Becton Dickinson CantolI flow cytometer using BD FACSDIVA software, and the results were analysed using Flowjo software (Tree Star Inc.). Cells were treated with $10 \mathrm{nM}$ Bromodeoxyuridine (BrdU) for $2 \mathrm{~h}$ prior to harvest by addition of trypsin. BrdU was detected using FITC-conjugated anti-BrdU (BD Bioscience) according to the manufacturer's protocol. Total DNA was detected by co-staining with propidium iodide (PI). For quantification of apoptosis, cells were assessed using the APC AnnexinV Apoptosis Detection Kit with PI (BioLegend, CA, USA; \#640932) as per the manufacturer's instructions. Between 5000 and 10,000 cells were analysed per sample and each experiment was conducted in triplicate.

\section{Patient-derived breast cancer xenograft (PDX) models}

All in vivo experiments, procedures and endpoints were approved by the Garvan Institute of Medical Research Animal Ethics Committee (protocol 15/25). KCC_P_3837 (HREC/13/RPA/187) was derived from an untreated grade 3, ER-positive, PR-positive, HER2negative primary invasive ductal carcinoma. Gar15-13 (\#14002, HREC/H2006/2633) was resected from a breast cancer liver metastasis of an endocrineresistant ER-positive, PR-negative, HER2-negative metastatic breast cancer [20]. At surgery, $4-\mathrm{mm}^{3} \mathrm{sec}-$ tions of tumour tissue were implanted into the 4th inguinal mammary gland of 6-8-week-old female NOD-SCID-IL2 $\gamma \mathrm{R}-/-$ mice (Australian BioResources Pty Ltd.) [21]. For KCC_P_3837, tumour growth was supported by implantation of a silastic pellet containing $0.72 \mathrm{mg} 17 \beta$-estradiol. Tumour growth was assessed twice weekly by calliper measurement and mice were randomised to treatment arms when tumours reached $150-250 \mathrm{~mm}^{3}$ (using the formula: width $^{2} \times$ length $\times 0.5$ ) using an online randomisation tool (https://www.graphpad.com/quickcalcs/randomize1.cfm). One hundred milligrammes/kilogramme NVP-CGM097 ( $20 \mathrm{mg} / \mathrm{ml}$ in $2 \%$ DMSO) was administered by oral gavage 5 days a week. Five milligrammes/body fulvestrant $(50 \mathrm{mg} /$ $\mathrm{ml}$ in peanut oil-Sigma P2144) was delivered by subcutaneous injection once per week. Mice were treated for 60 days or until tumour volume reached $1000 \mathrm{~mm}^{3}$.

\section{Immunohistochemistry (IHC) and quantification}

Tumour tissue was harvested and immediately fixed in $10 \%$ neutral buffered formalin at $4{ }^{\circ} \mathrm{C}$ overnight before dehydration and paraffin embedding. Antibodies used for IHC were anti-ER (M7047, 1:300, Agilent) and anti-Ki67 (M7240, 1:400, Agilent). Primary antibodies were detected using biotinylated IgG secondary antibodies (Agilent, 1:400), using streptavidin-HRP (Agilent) for amplification of signal followed by the 
addition of 3,3'-diaminobenzidine (Sigma) substrate. Images were scanned using Leica Aperio Slide Scanner (Leica Biosystems) and analysed using QuPath software to differentiate tumour tissue from stroma and necrosis, and to quantify $\mathrm{Ki}-67$ positivity in tumour tissue.

\section{Statistical analysis}

Statistical analyses were performed in GraphPad Prism (version 7.04, GraphPad Software) or in Microsoft Excel ( $x^{2}$ test for cell cycle phase analysis). ${ }^{*}=p<0.05,{ }^{* * *}=p<$ $0.01,{ }^{* * * * *}=p<0.001,{ }^{* * * * * *}=p<0.0001, \mathrm{~ns}=p>=0.05$.

\section{Differential expression and gene set enrichment analysis}

Total RNA was extracted from breast cancer cell lines and tissue samples using RNeasy Mini Kit (Qiagen) following the manufacturer's instructions. For tumour tissue, snap-frozen sections were first disrupted in lysis buffer in a TissueLyser II (QIAGEN). Sequencing data was processed on the Illumina HiSeq 2500 v4.0 System. Sequences were trimmed using Trim Galore (version 0.4.4) and genome-guided alignment to a human reference (HG38) was performed using STAR software (version 2.5). Feature counts were computed in $\mathrm{R}$ (version 3.4.3) using the package Rsubread [22]. Differential gene expression analysis was conducted with edgeR [23, 24] and limma [25] packages, on a threshold of $p . v a l<0.05$ and twofold change. The read count quantitation and normalised expression matrix can be downloaded from http://www.ncbi.nlm.nih.gov/ geo (GSE140758). Gene set enrichment analysis (GSEA) and pathway annotation were attained using cluterProfiler [26] in R. GSEA was tested on hallmark and curated gene sets and on gene ontology (GO) terms from the Molecular Signatures Database (MSigDB), Broad Institute (http://software.broadinstitute.org). Pathway enrichment was analysed in the Kyoto Encyclopedia of Genes and Genomes (KEGG) database (https://www.genome.jp/kegg/pathway.html).

\section{Results}

The small molecule MDM2 inhibitor NVP-CGM097 inhibits ER-positive breast cancer cells in a p53-dependent manner

ER-positive primary breast cancer has a relatively low incidence of TP53 mutation $(\sim 20 \%)$ [5]. To establish whether this situation is preserved in the resistant/ metastatic setting, we assessed the frequency of TP53 mutation or deletion across public cohorts in the cBio Cancer Genomics Portal (cBioPortal, http://cbioportal. org) (Fig. 1a). The incidence of TP53 aberration in advanced ER-positive breast cancers was $\sim 20 \%$, equivalent to that observed in primary tumours and relatively low compared to other cancer types
(International Agency for Research on Cancer, IARC) database (http://p53.iarc.fr). In the same clinical datasets, no instances of MDM2 mutation or deletion were detected (data not shown). Thus, the inhibition of MDM2 to increase p53 ${ }^{\text {wt }}$ activity could be a viable strategy in both primary and metastatic ER-positive breast cancer settings.

The MDM2 inhibitor NVP-CGM097 effectively hinders cancer cell proliferation in a $T P 53^{w t}$-dependent manner [27-29]. However, this has not been specifically demonstrated in ER-positive breast cancer models and we therefore determined the effect of NVP-CGM097 on cell viability in three in vitro models. After $48 \mathrm{~h}$ of treatment, TP5 $3^{w t}$ MCF-7 and TP53 ${ }^{w t}$ ZR75-1 responded to $1 \mu \mathrm{M}$ NVP-CGM097, exhibiting a loss of normal morphology with the adoption of stellate morphology and greater separation between cells (Fig. 1b). Submicromolar IC50 concentrations were measured by alamar blue assay for MCF-7, $1.78 \times 10^{-7} \mathrm{M}$ and ZR75-1, $2.05 \times 10^{-7} \mathrm{M}$ (Fig. 1c). TP53 mutant (TP53 ${ }^{\text {mut }}$ ) T-47D, however, showed no morphological changes after $48 \mathrm{~h}$ treatment with $1 \mu \mathrm{M}$ NVP-CGM097 (Fig. 1b) and exhibited an inhibitory response only at high doses (IC50, $\left.7.19 \times 10^{-6} \mathrm{M}\right)($ Fig. 1c).

\section{MDM2 inhibition activates p53 transcriptional activity in cell line models of ER-positive $\mathrm{p} 53^{\mathrm{wt}}$ breast cancer}

Using western blot analysis, we assessed p53 reactivation in cells treated with vehicle $(0.01 \%$ DMSO) or $1 \mu \mathrm{M}$ NVP-CGM097 for $48 \mathrm{~h}$ (Fig. 1d, Fig. S1A). In MCF-7 and ZR75-1, p53 levels increased in response to treatment, consistent with successful inhibition of MDM2mediated degradation of $\mathrm{p} 53$ protein. The $\mathrm{p} 53^{\mathrm{mut}}$ produced by T-47D accumulated in both vehicle and treated cells. Protein levels of the p53 transcriptional targets MDM2 and p21 were increased in MCF-7 and ZR75-1, but not in T-47D cell lines, after treatment. Collectively, these results indicate that NVP-CGM097 acts on-target to inhibit MDM2/p53 interaction with consequent $\mathrm{p} 53^{\mathrm{wt}}$ stabilisation and activation in models of ER-positive breast cancer.

We next performed RNA Sequencing (RNA Seq) on MCF-7 cell to interrogate effects on key p53 targets that are associated with cell cycle progression and programmed cell death (Fig. 1e). Treatment of MCF-7 cells with $1 \mu \mathrm{M}$ NVP-CGM097 for $48 \mathrm{~h}$ induced the expression of CDKN1A (p21), CCND1 (cyclin D1), SFN (14-33- $\sigma$ ) and GADD45A (Gadd45), which supports the activation of molecular mechanisms involved in cell cycle arrest. MDM2 inhibition also led to increased mRNA levels of apoptosis markers, including $F A S, B A X$ (Bax), $B I D$ (Bid) and $B B C 3$ (PUMA). These findings confirm NVP-CGM097-mediated p53 activation of downstream targets at the molecular level in MCF-7 cell lines. 


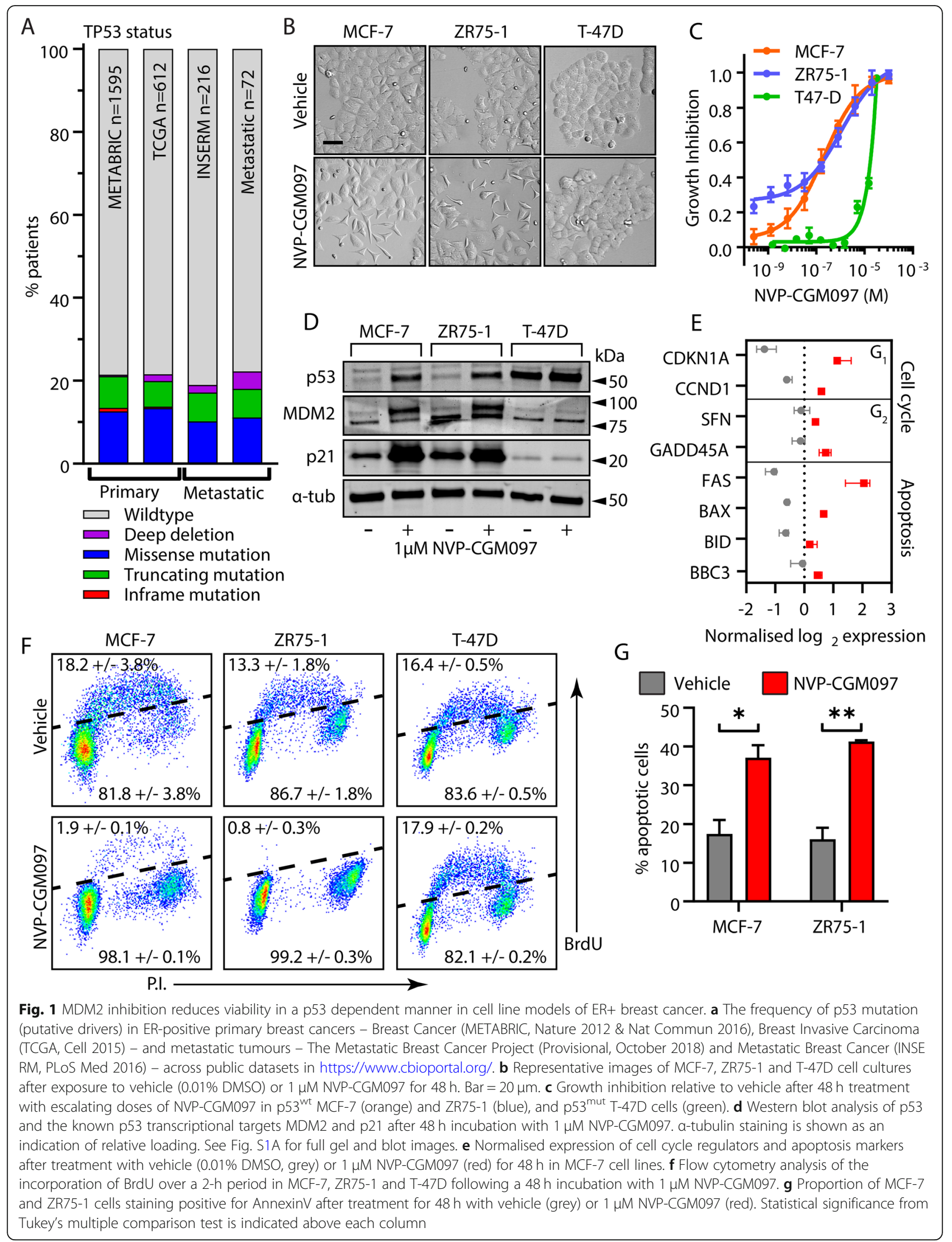


MDM2 inhibition causes $G_{1}$ and $G_{2} / M$ cell cycle arrest and apoptosis in treatment naïve breast cancer models

Given the several anti-proliferative outcomes of p53 activation, we next examined the mechanisms by which MDM2 inhibition compromises cell viability. Effects on cell cycle progression were assessed in MCF-7, ZR75-1 and T-47D cells after $48 \mathrm{~h}$ in the presence or absence of $1 \mu \mathrm{M}$ NVP-CGM097. We quantified total DNA content of individual cells and the incorporation of Bromodeoxyuridine (BrdU) over a 2-h pulse (Fig. 1f). In both MCF-7 and ZR75-1, treatment with NVP-CGM097 almost completely eliminated BrdU incorporation $\left(\chi^{2} p<\right.$ 0.001) with concomitant decrease in the proportion of cells in $\mathrm{S}$ phase and an increase in the proportion of cells in $\mathrm{G}_{2} / \mathrm{M}\left(\mathrm{MCF}-7 \chi^{2} p=0.003\right.$; ZR75- $1 \chi^{2} p<0.001$ ) (Fig. $\mathrm{S1B})$. In T-47D cells, there was no significant effect on either the incorporation of $\operatorname{BrdU}\left(\chi^{2} p=0.70\right)$ or on the distribution of cells in the cell cycle $\left(\chi^{2} p=0.78\right)$ after treatment with NVP-CGM097. We quantified the effect of NVP-CGM097 on apoptosis in MCF-7, ZR75-1 and T47D by staining for phosphatidylserine on the cell surface using fluorescent AnnexinV. Treatment with $1 \mu \mathrm{M}$ NVPCGM097 for $96 \mathrm{~h}$ significantly increased the proportion of apoptotic cells compared to vehicle-treated populations in both MCF-7 and ZR75-1 (MCF-7 $p<0.01$; ZR75-1 $p<$ 0.001) (Fig. 1g).

The effects of MDM2 inhibition were further validated on a $553^{\mathrm{wt}}$ patient-derived xenograft (PDX) model of endocrine-sensitive ER-positive breast cancer derived from a surgically resected primary treatmentnaïve ER-positive breast cancer (KCC_P_3837). Tumours were implanted concurrently with estradiol pellets and once tumour volume reached 100-150 $\mathrm{mm}^{3}$ were treated with vehicle (estradiol, 2\% DMSO) or NVP-CGM097 (100 mg/kg by daily gavage) for 60 days. NVP-CGM097 significantly inhibited tumour growth (Fig. 2a) and final tumour volume (Fig. S1C) relative to vehicle. Immunohistological quantification of the proliferation marker $\mathrm{Ki}-67$ at endpoint showed a significant decrease in the proportion of Ki-67positive cells in tumours treated with NVP-CGM097 (Fig. 2b, Fig. S1D). Together, these data show that NVP-CGM097 is an effective inhibitor of proliferation in breast cancer models via p53-dependent induction of apoptosis and cell cycle arrest.

\section{The combination of NVP-CGM097 and fulvestrant synergistically inhibits MCF-7 cells without increasing apoptosis}

Fulvestrant is a standard therapeutic option in the treatment of women with advanced ER-positive breast cancer. We therefore combined NVP-CGM097 with fulvestrant and measured viability of parental MCF-7 cells to test for an enhanced effect through drug additivity or synergy (Fig. 3a). We calculated combination indices using Compusyn software and detected a significant synergistic effect $\left(\log _{2}\right.$ combination index $<0)$ on viability when combining the drugs (Fig. 3b). We then quantified the effect of NVP-CGM097 alone and in combination with fulvestrant on the rate of apoptosis in MCF-7 cells (Fig. 3c). As previously shown (Fig. 1f), treatment with $1 \mu \mathrm{M}$ NVP-CGM097 for $96 \mathrm{~h}$ significantly increased the proportion of apoptotic cells compared to vehicle-treated

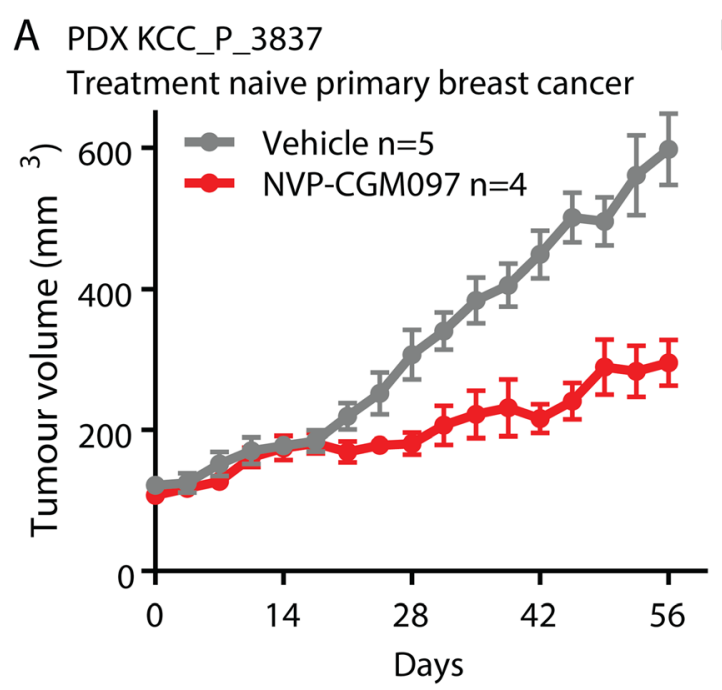

B

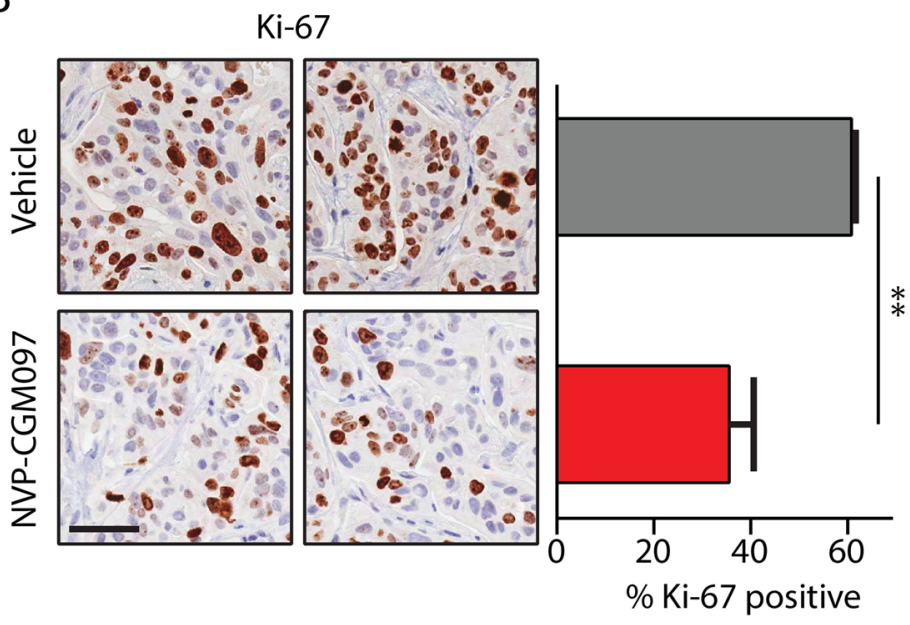

Fig. 2 MDM2 inhibition reduces tumour growth and proliferation markers in an ER+ breast cancer PDX model. a Growth of the treatment naïve KCC_P_3837 PDX model of ER+ breast cancer treated with vehicle (2\% DMSO daily, grey, $n=5)$ and NVP-CGM097 (100 mg/kg daily, red, $n=4)$ for 60 days. b Representative images and quantification of the proliferation marker Ki-67 at endpoint. Ki-67 positive proportions from three independent replicates per treatment were compared by two-tailed $T$ test. Bar $=50 \mu \mathrm{m}$ 


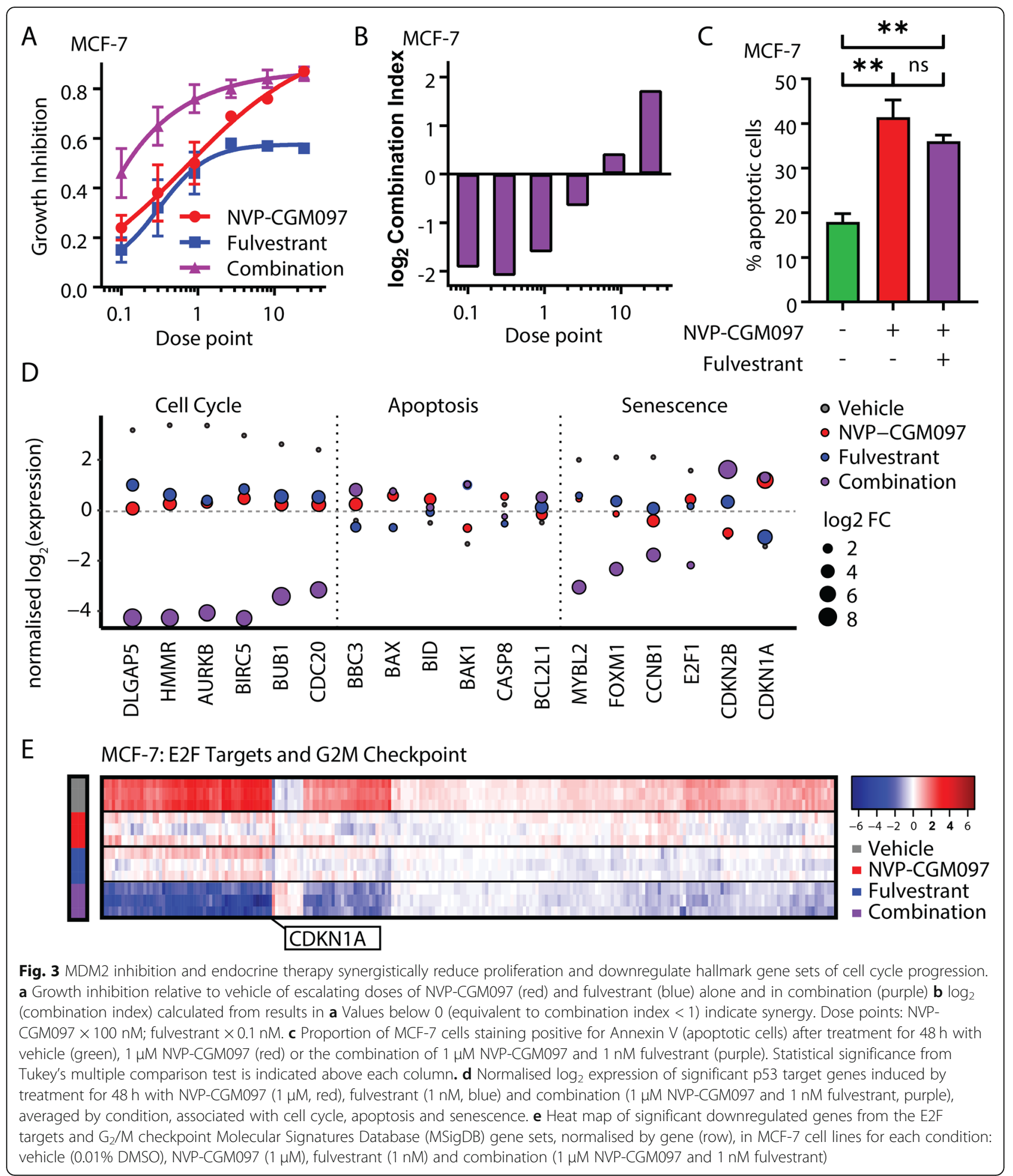

populations $(p<0.01)$. By contrast, the inclusion of 1 $\mathrm{nM}$ fulvestrant yielded no significant additional effect, suggesting that the synergy observed in the viability assays does not occur via enhancement of p53mediated apoptosis.
Combination of NVP-CGM097 with fulvestrant downregulates $\mathrm{E} 2 \mathrm{~F}$ and $\mathrm{G}_{2} / \mathrm{M}$ transcriptional programmes in vitro

To identify the molecular mechanisms underlying the combination effects of NVP-CGM097 and fulvestrant, 
we quantified the transcriptional profile of MCF-7 cells after $48 \mathrm{~h}$ treatment with $0.01 \%$ DMSO, $1 \mu \mathrm{M}$ NVPCGM097, $1 \mathrm{nM}$ fulvestrant or the combination of NVPCGM097 and fulvestrant at these doses (Fig. S2A). Under stringent thresholds (adjusted $p$ value $<0.05$ and 2 -fold change), 576 genes were significantly regulated by NVP-CGM097 (Table S1), 803 by fulvestrant (Table S2) and 1104 by combination (Table S3) compared to vehicle. Combination therapy incorporated most of NVPCGM097 (80\%) and fulvestrant (90\%)-induced gene regulation and further altered unique gene sets that are central players in DNA replication, including the minichromosome maintenance protein complex (MCM) and DNA polymerase subunits (Fig. S2B, Table S4). The involvement of these additional gene sets suggests direct and indirect downstream regulation of the cell cycle machinery.

Kyoto Encyclopedia of Genes and Genomes (KEGG) pathway enrichment analysis of differential gene expression induced by combination therapy revealed a significant negative regulation of Cell Cycle $(p=1.08 \mathrm{E}-21$; Fig. S2C) and positive enrichment of p53 Signalling Pathway $(p=1.95 \mathrm{E}-10$; Fig. S2, C and D; Table S5) compared to vehicle. NVP-CGM097 in combination with fulvestrant significantly regulated a set of genes involved in intertwined signalling cascades of cell cycle, apoptosis and cellular senescence (Fig. 3d; Fig. S3A). In MCF-7 cell lines, combination therapy enhanced the downregulation of essential components of the cell cycle (HMMR, $A U R K B, B I R C 5)$ that control proliferative activities and tumour growth alongside downregulation of the antisenescence markers FOXM1 and E2F1 [30]. FOXM1 also regulates the expression of $\mathrm{G}_{2} / \mathrm{M}$ cell cycle genes, including the levels of its known mitotic targets CCNB1 and $C D C 20$ [31, 32]. The upregulation of prosenescence markers CDKN1A (p21) and CDKN2B (p15)-master effectors of multiple tumour suppressor pathways-and pro-apoptotic markers, such as $B B C 3$, $B A X, B I D$ and $B A K 1$, also contributes to the regulation of cell homeostasis, although our in vitro data of combination therapy indicate that synergy between treatments is not dependent on increased apoptosis.

Gene set enrichment analysis (GSEA) revealed enhanced downregulation of key hallmarks involved in cell proliferation and cell cycle regulation, including E2F Targets and $G_{2} / M$ Checkpoint, in combination treated cells compared to either drug alone (Fig. 3e). To quantify the additional effect elicited by combination therapy at the transcriptional level, we computed a score for each gene $x$ based on the log fold change compared to vehicle for each condition (Tables S1-3) as follows: Score $_{x}=\log$ $2\left(\right.$ Combination $_{x} /\left(\right.$ NVPCGM097 $_{x}+$ fulvestrant $\left.\left._{x}\right)\right) \quad$ (Table S6). Using this formula, a score greater than 0 denotes transcripts in which the differential gene expression detected for the combination treatment exceeds the sum of effect for each individual treatment. Transcripts from the KEGG annotated Cell Cycle gene set were significantly over-represented within the cohort scoring above 0 (Wilcoxon rank sum test $p=4.45 \times 10^{-7}$ ).

\section{The combination of MDM2 inhibition and fulvestrant increases senescence in fulvestrant-resistant cells compared to MDM2 inhibition alone}

We next asked whether combining NVP-CGM097 and fulvestrant could lead to enhancement or re-sensitisation to therapy in fulvestrant-resistant MCF-7 (FasR) cell lines. MCF-7 FasR were generated by the continuous administration of $100 \mathrm{nM}$ fulvestrant for 12 months to MCF-7 cells. Resistance was confirmed by examination of relative proliferation of the parental MCF-7 cells and MCF-7 FasR cells using an Incucyte confluence assay (Fig. S4A). We first treated MCF-7 FasR cells with escalating doses of NVP-CGM097 in the presence or absence of $100 \mathrm{nM}$ fulvestrant (the dose used to generate fulvestrant resistance in this model) (Fig. 4a). No significant difference was observed in cell viability between the two treatments using the alamar blue assay. Treatment with $1 \mu \mathrm{M}$ NVP-CGM097 for $48 \mathrm{~h}$ significantly altered the cell cycle phase distribution of MCF-7 FasR cells $\left(x^{2} p<\right.$ $0.001)$ resulting in a decrease in the proportion of cells in $\mathrm{S}$ phase, consistent with our findings from parental MCF-7 cells, and no significant difference in distribution was observed with the addition of $100 \mathrm{nM}$ fulvestrant (Fig. 4b). Increased levels of p21 protein were comparable between treatment with $1 \mu \mathrm{M}$ NVP-CGM097 alone for $48 \mathrm{~h}$ and after treatment in combination with 100 nM fulvestrant (Fig. 4c, Fig. S4B). Treatment with 100 $\mathrm{nM}$ fulvestrant alone yielded no change in p21 levels compared to untreated cells. We observed phenotypically distinct cells following treatment with NVPCGM097 alone and in combination with fulvestrant, with a flattened morphology reminiscent of senescence (Fig. 4d). Consequently, we analysed the cells for senescence via accumulation of senescence-associated $\beta$ galactosidase. We observed a significant increase in $\beta$ galactosidase staining in cells treated with the combination of $1 \mu \mathrm{M}$ NVP-CGM097 and $100 \mathrm{nM}$ fulvestrant for $72 \mathrm{~h}$ compared to vehicle treatment or treatment with either single agent alone ( $p<0.01$ vs NVP-CGM097; $p<$ 0.001 vs fulvestrant) (Fig. 4e).

\section{Combination NVP-CGM097/fulvestrant reduces tumour growth in an in vivo PDX model of endocrine resistance}

To investigate this enhancement of effect in vivo, we treated a p53 ${ }^{\mathrm{wt}}$ PDX model of endocrine-resistant ERpositive breast cancer, Gar15-13 [20], with vehicle (DMSO), NVP-CGM097, fulvestrant or combination therapy. Gar15-13 was established from a liver 
A

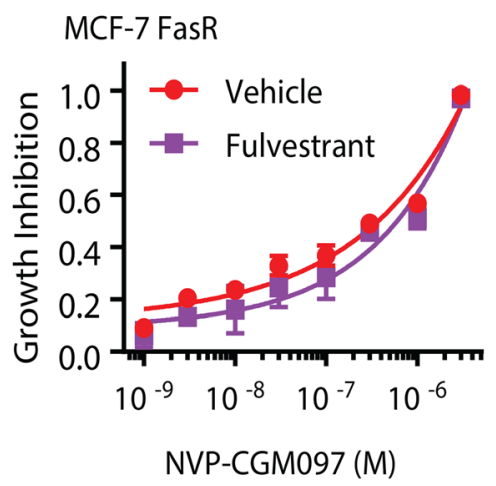

C

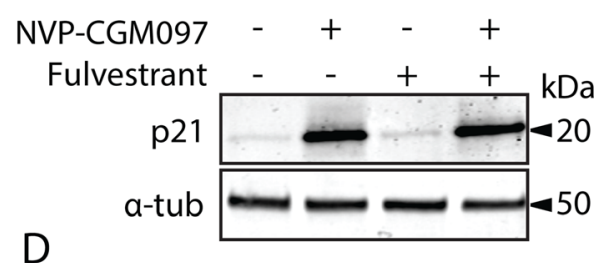

$\beta$-galactosidase staining

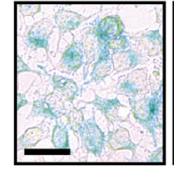

Vehicle

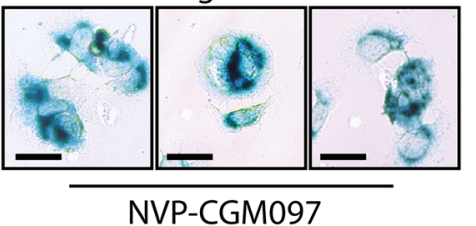

B

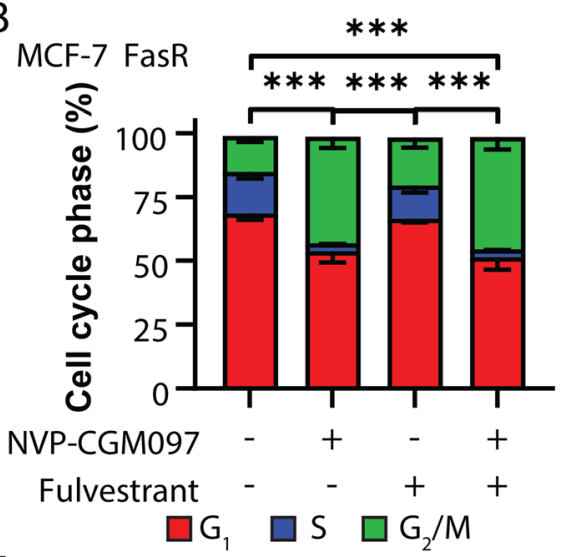

E

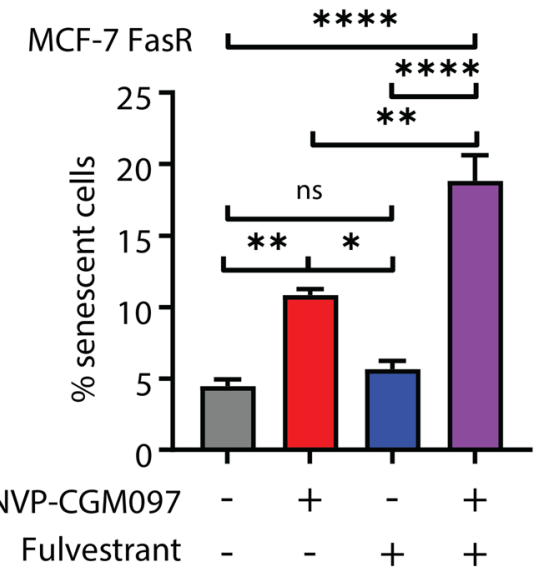

Fig. 4 Fulvestrant potentiates MDM2 inhibition in a fulvestrant-resistant cell line model. a Growth inhibition of MCF-7 FasR relative to vehicle with escalating doses of NVP-CGM097 in the presence (purple) or absence (red) of $100 \mathrm{nM}$ fulvestrant-the dose used to generate the resistant cell line. No significant difference was observed after $48 \mathrm{~h}$ of treatment. $\mathbf{b}$ Flow cytometric quantification of propidium iodide incorporation into DNA compared to vehicle in MCF-7 FasR after incubation for $48 \mathrm{~h}$ with vehicle (0.01\% DMSO), 1 MM NVP-CGM097, $100 \mathrm{nM}$ fulvestrant, or the combination of both drugs. Statistical significance from $x^{2}$ test using the vehicle-treated profile as the expected value is indicated. Red $=G_{1}\left(\right.$ bottom), blue $=S$ (middle), green $=G_{2} /$ M (top). c Western blot analysis of the p53 transcriptional target p21 in MCF-7 FasR cells after $48 \mathrm{~h}$ incubation with $1 \mu$ M NVP-CGM097. a-tubulin staining is shown as an indication of relative loading. See Fig. S4B for full gel and blot images. $\mathbf{d}$ Representative images of MCF-7 FasR cell cultures after exposure to vehicle $(0.01 \%$ DMSO) or $1 \mu \mathrm{M}$ NVP-CGM097 for $48 \mathrm{~h}$ followed by staining for senescence-associated $\beta$-galactosidase. Bar $=20 \mu \mathrm{m}$ e Quantification of MCF-7 FasR cells staining positive for senescence-associated $\beta$-galactosidase activity after treatment for $48 \mathrm{~h}$ with vehicle $(0.01 \%$ DMSO, grey), 1 MM NVP-CGM097 (red), 100 nM fulvestrant (blue) or the combination of NVP-CGM097 and fulvestrant (purple). Statistical significance from Tukey's multiple comparison test is indicated above each column

metastasis progressing on the aromatase inhibitor anastrozole. It maintains high ER positivity but grows independently of estradiol supplementation, consistent with its prior exposure to aromatase inhibition in the patient [20]. This model is resistant to fulvestrant and fulvestrant-treated tumours progressed rapidly to tumour volume endpoint with growth kinetics and survival outcomes similar to the vehicle arm (Fig. 5a). NVPCGM097 alone significantly inhibited tumour growth (Fig. 5a) and extended survival (Fig. 5b, c) and surprisingly, given the model's resistance to fulvestrant, combination treatment reduced tumour growth (Fig. 5a) and increased survival time significantly more than NVPCGM097 alone, with benefit continuing beyond the 6- week period of administration (Fig. 5b, c). Gar15-13 PDX treated for 10 days with NVP-CGM097 alone or in combination with fulvestrant showed reduced Ki-67 staining compared to vehicle-treated tumours, although no significant difference was observed between the NVP-CGM097 only arm and the combination arm (vehicle vs NVP-CGM097 $p<0.01$; vehicle vs combination $p=0.011$, all other pairwise comparisons not significant at $\alpha=0.05$ ) (Fig. 5d). Tumours treated for 10 days with fulvestrant (either alone or in combination) showed a significant reduction in the intensity of ER staining by immunohistochemistry in both the nucleus and cytoplasm (vehicle vs fulvestrant-nuclear ER $p=0.038$, cytoplasmic ER $p<0.01$; vehicle vs combination- 

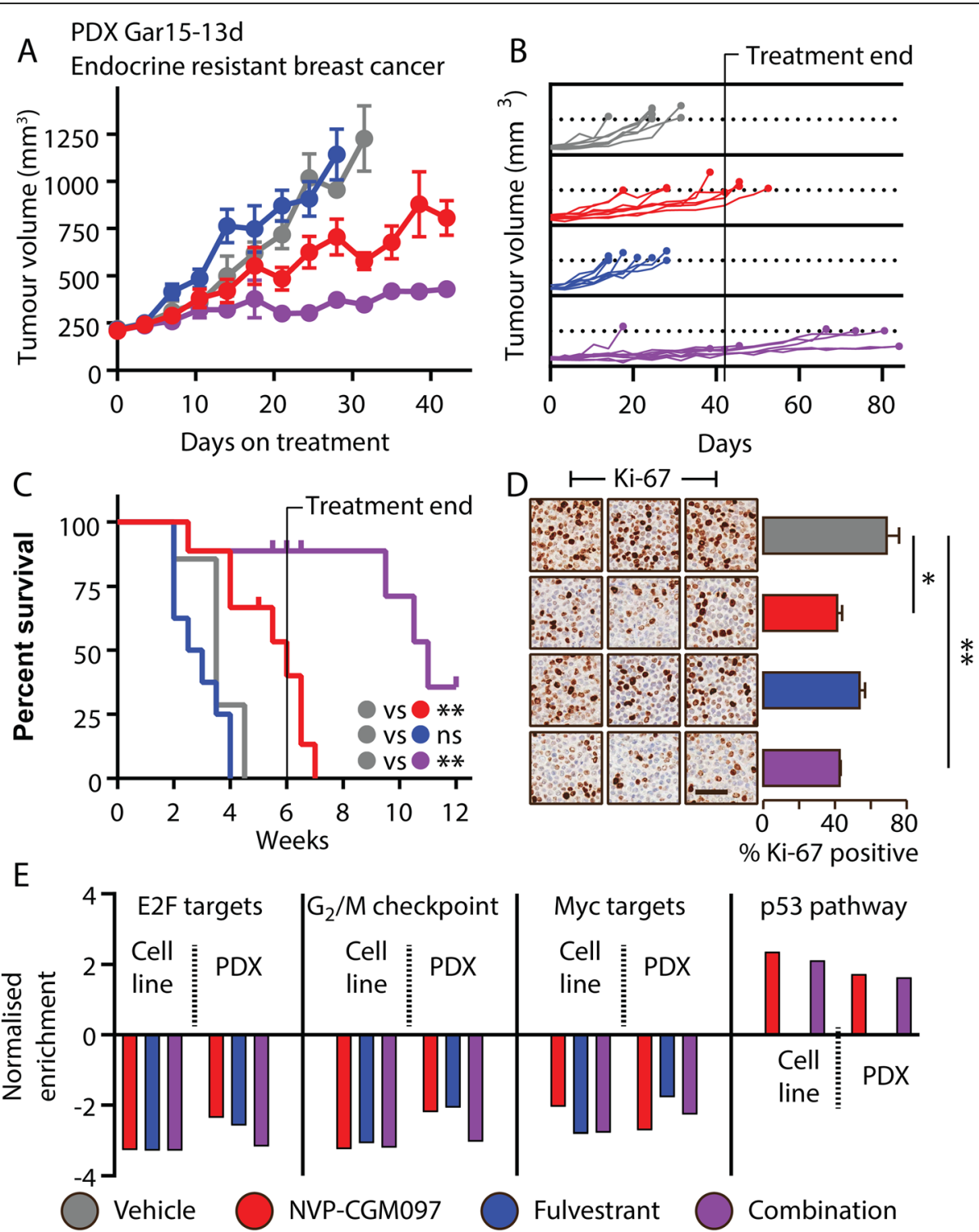

Fig. 5 Fulvestrant potentiates MDM2 inhibition in vivo in a PDX model of fulvestrant-resistant ER+ breast cancer. a Growth of the endocrine-resistant Gar15-13 PDX model of ER+ breast cancer treated with vehicle (2\% DMSO daily, grey, $n=7$ ), NVP-CGM097 (100 mg/kg daily, red, $n=8)$, fulvestrant (5 mg/ body weekly, blue, $n=8$ ) or the combination of NVP-CGM097 and fulvestrant (purple, $n=9$ ). $\mathbf{b}$ Continued growth of tumours following withdrawal of treatment after 6 weeks. c Survival analysis of outcomes after treatment with combination therapy compared to each drug alone (an event was defined as a tumour exceeding $999 \mathrm{~mm}^{3}$ ). Curves were compared using Mantel-Cox log-rank test and significance is indicated for comparisons of each arm with vehicle. $\mathbf{d}$ Representative images and quantification of $\mathrm{IHC}$ staining for Ki-67 of three independent replicates per treatment arm from tumours treated for 10 days. Statistical significance from Tukey's multiple comparison test is indicated. Bar $=50 \mu \mathrm{m}$. e GSEA analysis compared to vehicle performed across gene expression sets of in vitro and in vivo models following treatment with NVP-CGM097 (red), fulvestrant and combination therapy show consistent regulation of hallmark signatures from MSigDB: E2F targets, $\mathrm{G}_{2} / \mathrm{M}$ checkpoints, MYC targets and p53 pathway. This analysis is reinforced by the normalised enrichment score (NES) that shows the difference in gene set size following each treatment conditions

nuclear ER $p=0.013$, cytoplasmic ER $p<0.001$ ) (Fig. S4C), demonstrating efficacy of fulvestrant in targeting ER but not in directly inhibiting growth. The observed enhancement of the effects of NVP-CGM097 with the addition of fulvestrant may therefore be the result of an interaction with functions of ER not directly associated with proliferation, for example, in the context of senescence as we have demonstrated in MCF-7 FasR.
Effects of single-agent and combination therapy reconcile targeted molecular mechanisms in both in vitro and in vivo models

RNASeq analysis of short-term (10 day) treated tumours showed differential genes $(p<0.05)$ induced by NVPCGM097 $(n=141)$, fulvestrant $(n=411)$ and combination therapy $(n=484)$ (Table S7-9). GSEA demonstrated negative enrichment of E2F targets and $\mathrm{G}_{2 /} \mathrm{M}$ 
checkpoint and other relevant hallmark gene sets, including MYC targets and p53 pathway (Table S1015). These signatures were concurrently enriched in both in vitro and in vivo models (Fig. 5e). In Gar1513, combination therapy elicited an enhanced effect on cell cycle proliferative gene sets, E2F and $G_{2} / M$ (Fig. S4D and E; Table S16) compared to single-agent treatments. Notably, fulvestrant treatment in vitro or in vivo did not show an effect on the hallmarks of p53 pathway (Fig. 5e), which was reassuringly strongly upregulated by treatment with NVP-CGM097 in both settings.

Combination treatment of NVP-CGM097 with fulvestrant also led to decreased expression of key cell cycle markers, including DLGAP5, HMMR, AURKB, BIRC5, $B U B 1$ and $C D C 20$ (Fig. S4E), which is consistent with those identified in MCF-7-treated cells. The strong inhibitory effect of combination therapy on cell cycle thus provide a preclinical rationale to use MDM2 inhibitors with ER degraders to enhance the therapeutic effects of endocrine sensitive and resistant ER-positive $\mathrm{p} 53^{\mathrm{wt}}$ breast cancer.

\section{MDM2 inhibition synergises with CDK4/6 inhibitors via the same mechanisms that underlie synergy with fulvestrant}

Treatment with CDK4/6 inhibitors has become a standard of care for advanced ER-positive breast cancer that has failed endocrine therapy [4]. CDK4/6 inhibitors act through the $G_{1} / S$ axis to prevent cell cycle entry and have many overlapping target genes with endocrine therapy. Consequently, we investigated the potential of combination therapy of NVP-CGM097 and palbociclib, a major CDK4/6 inhibitor used in clinical practice. We performed a constant dose ratio protocol with NVPCGM097 and palbociclib (Fig. 6a), followed by

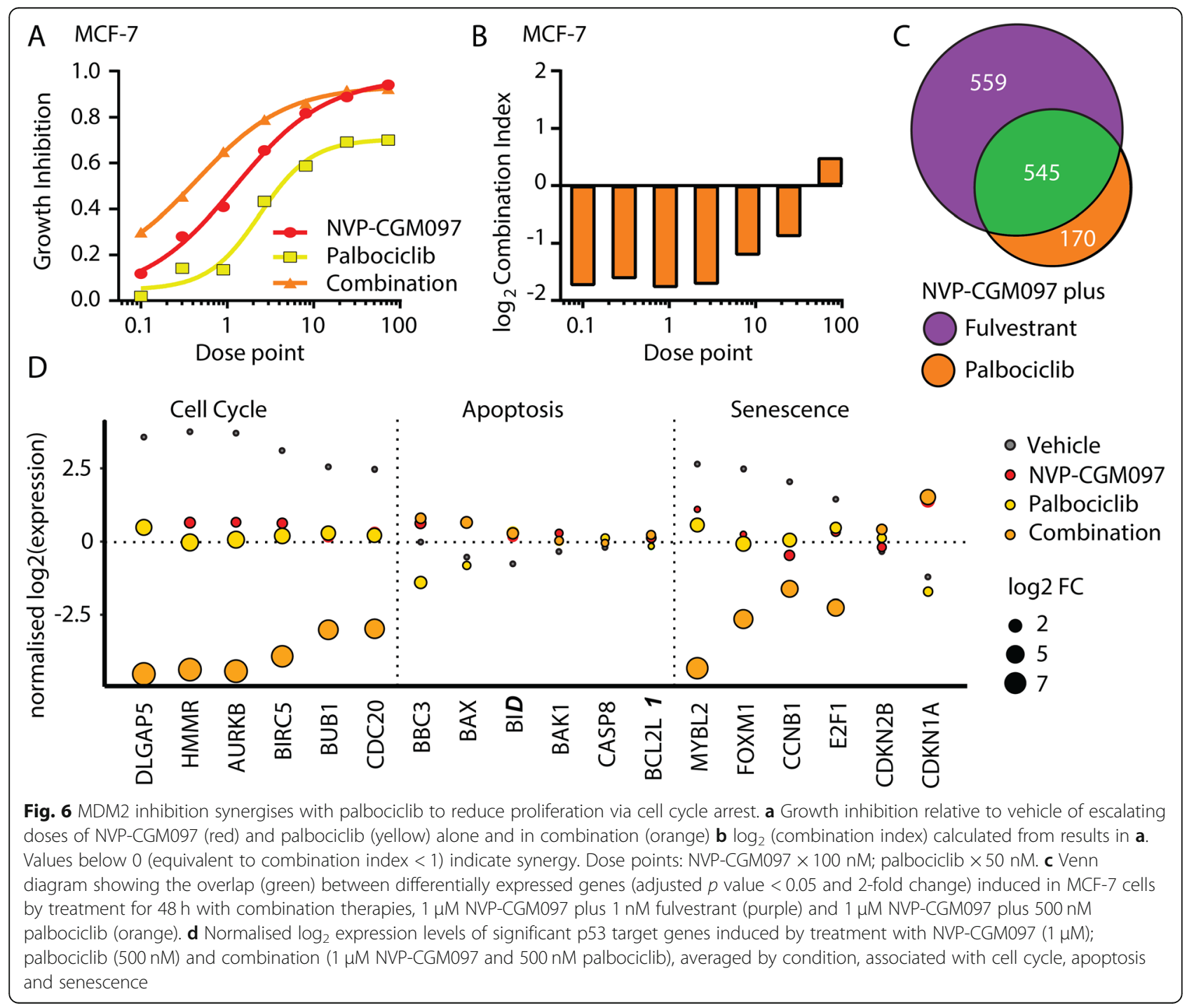


combination index analysis on MCF-7 cells. A significant synergistic effect on viability was observed with the combination therapy compared to either single agent alone (Fig. 6b).

We next investigated the combinatorial effect of NVPCGM097 and palbociclib by RNA-Seq (Fig. S5A). We confirmed significant (adjusted $p$ value $<0.05$ and 2-fold change) gene expression changes induced by NVPCGM097 $(n=572)$, palbociclib $(n=392)$ and combination therapy $(n=714)(S 17-19)$, compared to vehicle (0.01\% DMSO). Combination therapy encompassed about $90 \%$ of the changes promoted by either agent alone (Fig. S5B). Combination of NVP-CGM097 with fulvestrant or with palbociclib showed a significant overlap (Fig. 6c). As with NVP-CGM097 plus fulvestrant, when we quantified the additional effect elicited by combination therapy at the transcriptional level (Table S20), transcripts from the KEGG annotated Cell Cycle gene set were significantly over-represented within the cohort scoring above 0 (Wilcoxon rank sum test $p=1.52 \times$ $10^{-13}$ ). Combination of NVP-CGM097 with palbociclib further downregulates the senescence gatekeepers MYBL2, FOXM1, CCNB1 and E2F1 (Fig. 6d). Although both fulvestrant and palbociclib interact with the p53 pathway at numerous points, especially fulvestrant which ablates the interaction between ER and p53 [14], our data suggest that the major common mechanistic events underlying the efficacy of the two combination strategies with NVP-CGM097 are related to the activity of each drug in terms of inhibiting cell cycle progression.

\section{The combination of MDM2 inhibition and palbociclib increases senescence in palbociclib-resistant cells compared to MDM2 inhibition alone}

We investigated the effect of MDM2 inhibition on MCF-7 cells with acquired resistance to palbociclib (PalbR). MCF-7 PalbR were generated by the continuous administration of $500 \mathrm{nM}$ palbociclib for 6 months to MCF-7 cells. Resistance was confirmed by examination of relative growth inhibition of the parental MCF-7 cells and MCF-7 PalbR cells to a range of doses of palbociclib (Fig. S5C). NVP-CGM097 inhibited the growth of MCF7 PalbR cells with an IC50 of $9.0 \times 10^{-8}$ M $(95 \%$ C.I. $6.7 \times 10^{-8} \mathrm{M}$ to $1.1 \times 10^{-7} \mathrm{M}$ ), as determined by alamar blue assay (Fig. 7a). Analysis of cell cycle phase by flow cytometry showed a significant difference $\left(\chi^{2} p<0.001\right)$ in distribution from vehicle $(500 \mathrm{nM}$ palbociclib, $0.01 \%$ DMSO) following treatment for $48 \mathrm{~h}$ with $1 \mu \mathrm{M}$ NVPCGM097 (Fig. 7b) with an increase in the proportion of cells in $G_{2} / M$. A key difference to endocrine resistance models was that MCF-7 PalbR cells did not accumulate in $G_{1}$ after NVP-CGM097 treatment. This suggests that cells resistant to CDK4/6 inhibition may be able to overcome the $G_{1}$ block associated with activation of p53 to some extent but are still susceptible to cell cycle inhibition in $\mathrm{S}$ and/or $\mathrm{G}_{2} / \mathrm{M}$. Treatment with $1 \mu \mathrm{M}$ NVPCGM097 for $96 \mathrm{~h}$ resulted in a significant increase in the proportion of apoptotic cells compared to vehicle $(0.01 \%$ DMSO) or $500 \mathrm{nM}$ palbociclib-treated cells $(p<0.001)$ but the combination treatment yielded no significant increase compared to NVP-CGM097 alone (Fig. 7c).

We had observed that NVP-CGM097 in combination with fulvestrant resulted in increased senescence compared to NVP-CGM097 alone in fulvestrant-resistant cells. We examined whether a similar increase could also be observed with CDK4/6 inhibitor-resistant cells treated with combination NVP-CGM097 and palbociclib. MCF-7 PalbR cells were treated with $1 \mu \mathrm{M}$ NVPCGM097 in combination with $500 \mathrm{nM}$ palbociclib (the resistance dose) for $48 \mathrm{~h}$. Treatment with either singleagent NVP-CGM097 or the combination with palbociclib increased expression of the senescence marker p21 [33] (Fig. 7d, Fig. S5D) and there was a significant increase in the proportion of $\beta$-galactosidase positive cells in the combination treatment compared to either single agent alone ( $p<0.001$ vs NVP-CGM097 or palbociclib) (Fig. 7e, f). Thus, NVP-CGM097 is an effective inhibitor of ER-positive breast cancer even in the context of palbociclib resistance, and as with endocrine therapy, rechallenging cells in the presence of NVP-CGM097 increases the incidence of senescence compared with single-agent treatments.

\section{Discussion}

The treatment of patients with endocrine-resistant ERpositive breast cancer is extremely challenging, as in this setting, disease leads inevitably to death. Numerous mechanisms of treatment resistance have been identified. While some of these pathways are targetable, for instance, the PI3K pathway and the cyclinD-CDK4/6-Rb axis; the optimal next-line treatment strategies are unclear. In this context, MDM2 inhibition to reactivate p53 is particularly attractive in ER-positive breast cancer as it has a relatively low incidence of Tp53 mutation in the primary and metastatic setting $(\sim 20 \%)$ [5]. In addition, those ER-positive breast cancer patients who respond to CDK4/6 inhibitors are more likely to have intact p53 [34], meaning that there is the potential to improve the response to CDK4/6 inhibitors through p53 activation.

A number of small molecule MDM2 inhibitors have been designed and are at various stages of clinical development [35]. NVP-CGM097 was one of the first of the new generation inhibitors and has advanced to phase 1 clinical trial (NCT01760525). Although the final outcomes of the trial have not yet been reported, early results showed clinical activity-predominantly stable disease-and establishment of a tolerable dose regimen, with one quarter of patients receiving treatment for at 


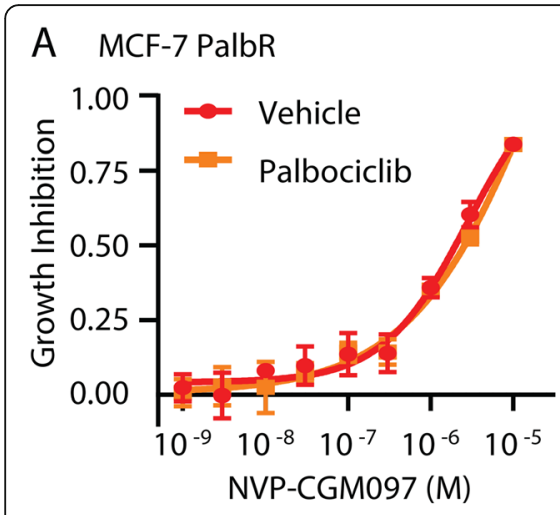

D

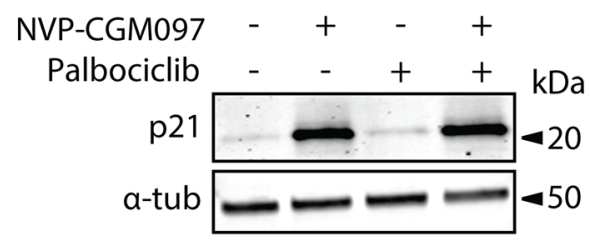

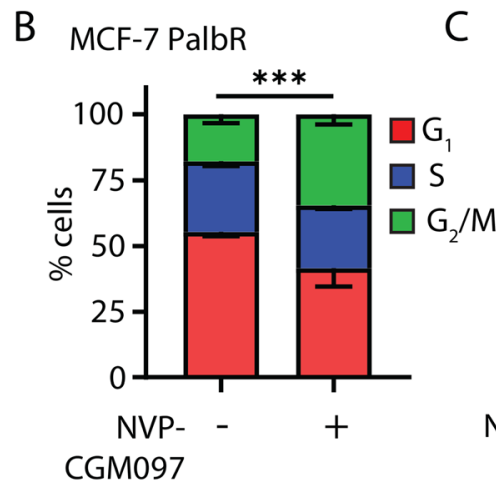

C MCF-7 PalbR
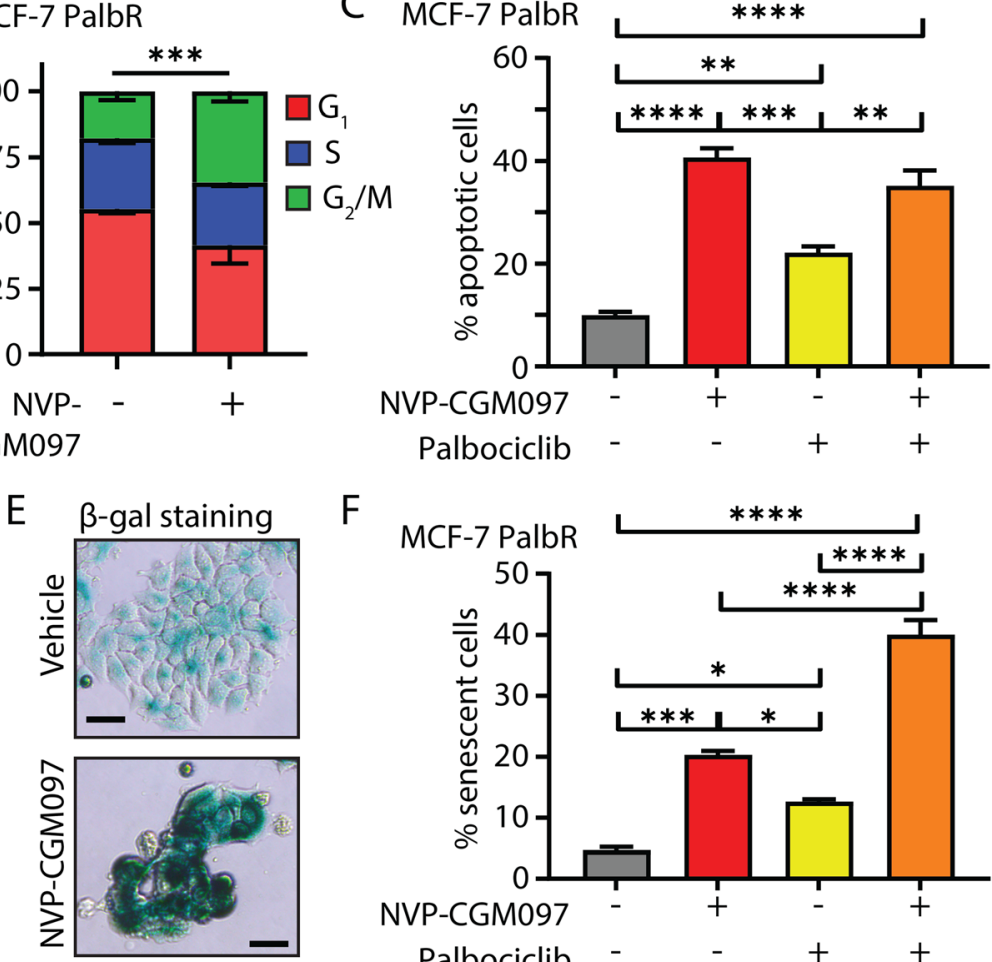

$\mathrm{F}$

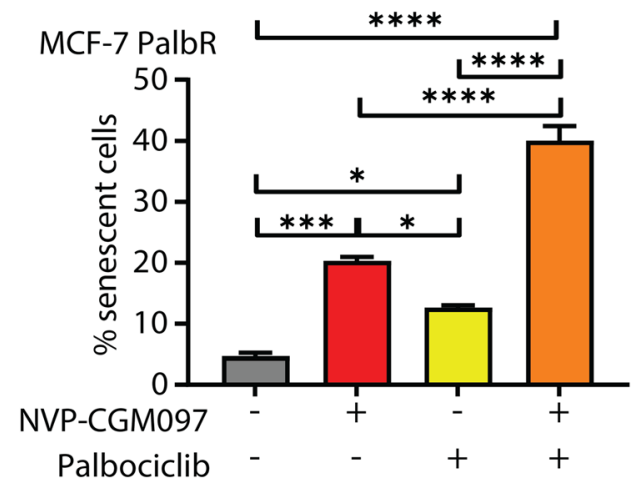

Fig. 7 Palbociclib potentiates MDM2 inhibition via an increase in senescence in palbociclib-resistant MCF-7. a Growth inhibition of MCF-7 PalbR relative to vehicle $(0.01 \%$ DMSO) of escalating doses of NVP-CGM097 in the presence (red) or absence (yellow) of $500 \mathrm{nM}$ palbociclib-the dose used to generate the resistant cell line. No significant difference was observed after $48 \mathrm{~h}$ of treatment. b Proportion of cells in each cell cycle phase from flow cytometric quantification of propidium iodide staining of genomic DNA in MCF-7 PalbR cells after $48 \mathrm{~h}$ treatment with $1 \mu \mathrm{M}$ NVP-CGM097 compared to vehicle (500 nM palbociclib, 0.01\% DMSO). Statistical significance from $x^{2}$ test using the vehicle-treated profile as the expected value is indicated. Red $=G_{1}$ (bottom), blue $=S$ (middle), green $=G_{2} / M$ (top). c Proportion of MCF-7 PalbR cells staining positive for AnnexinV after treatment for $48 \mathrm{~h}$ with vehicle (0.01\% DMSO, grey), $1 \mu \mathrm{M}$ NVP-CGM097 (red), $500 \mathrm{nM}$ palbociclib (yellow) or the combination of $1 \mu \mathrm{M}$ NVP-CGM097 and $500 \mathrm{nM}$ palbociclib (orange). Statistical significance from Tukey's multiple comparison test is indicated above each column. $\mathbf{d}$ Western blot analysis of p21 in MCF-7 PalbR cells after $48 \mathrm{~h}$ incubation with $1 \mu$ M NVP-CGM097. a-tubulin staining is shown as an indication of relative loading. See Fig. S5D for full gel and blot images. e Representative images of MCF-7 PalbR cell cultures after exposure to vehicle (500 nM palbociclib, 0.01\% DMSO) or $1 \mu \mathrm{M}$ NVP-CGM097 for $48 \mathrm{~h}$ followed by staining for senescence-associated $\beta$-galactosidase. Bar = $20 \mu \mathrm{m}$. $\mathbf{f}$ Quantification of MCF-7 PalbR cells staining positive for senescence-associated $\beta$-galactosidase activity after treatment for $48 \mathrm{~h}$ with vehicle (0.01\% DMSO, grey), 1 MM NVP-CGM097 (red), 500 nM palbociclib (yellow) or the combination of NVP-CGM097 and palbociclib (orange). Statistical significance from Tukey's multiple comparison test is indicated above each column

least 24 weeks [18]. The reported phase I dose of CGM097 was 300-700 mg three times per week on a 21-day cycle with 2 weeks on and 1 week off. p53 pathway activation was demonstrated by the induction of its downstream target GDF-15 in serum and plasma. This aligns well with the dose used in this study of $100 \mathrm{mg} / \mathrm{kg}$ in mice, which converts to a human equivalent dose of $\sim 500 \mathrm{mg}$ for a $60-\mathrm{kg}$ patient [36]. As with most MDM2 inhibitors that have so far reached clinical trials, side effects of NVP-CGM097 include thrombocytopenia and neutropenia which would need to be considered in any clinical application in combination with drugs with overlapping toxicity profiles such as CDK4/6 inhibitors [37]. Importantly, our data show that combination of NVPCGM097 with fulvestrant, which is not associated with haematological adverse effects, has a highly similar response profile to combination with CDK4/6 inhibition in terms of the key molecular pathways affected. It is currently unclear as to whether NVP-CGM097 itself will continue in development; however, a range of nextgeneration MDM2 inhibitors with improved tolerability are in development [38]. Our results demonstrate that MDM2 inhibition is a rational target for novel therapeutic strategies in the setting of treatment-resistant breast cancer.

In this study, we show that reactivation of wildtype p53 using NVP-CGM097 is an effective inhibitor of tumour growth in multiple models of endocrine-naïve, endocrine-resistant and CDK4/6 inhibitor-resistant ERpositive breast cancer in vitro and in vivo. We identify the key mechanisms of the synergistic interactions between NVP-CGM097 and endocrine therapy, which 
occurs through the inhibition of E2F targets and $\mathrm{G}_{2} / \mathrm{M}$ checkpoint signalling and induction of senescence, rather than via a general upregulation of p53-dependent apoptotic pathways above the level of MDM2 inhibition alone. Moreover, we find these same pathways are specifically targeted in response to combined inhibition of both MDM2 and the CDK4/ 6 pathway.

Consistent with activation of p53, NVP-CGM097 induced apoptosis, and cell cycle arrest in $G_{1}$ and $G_{2}$, in TP53 ${ }^{w t}$ ER-positive breast cancer cell line (MCF-7 and ZR75-1) models. The only setting in which we did not observe anti-tumour effect was in the case of the TP53 ${ }^{\text {mut }}$ T-47D cell line model which supports our conclusion that activity of NVP-CGM097 requires wildtype p53. MDM2 inhibition was characterised by very high expression of the key cell cycle regulator p21-which is a p53 target and principal component of cell cycle arrest in $G_{1}$ and $G_{2}$ and in the induction and maintenance of senescence [39].

Our data from ER-positive PDX models shows that NVP-CGM097 is effective as a single agent, and it combines with fulvestrant to be highly effective in the fulvestrant-resistant Gar15-13 PDX model. In the only other breast cancer PDX experiments so far reported, another MDM2 inhibitor MI-77301 was effective at reducing tumour growth of two fulvestrant-resistant PDX models [16]. Similar to our findings, co-administration of fulvestrant led to prolonged therapeutic benefit after treatment cessation in one model, although unlike our model no additional benefit was observed during treatment. The second PDX model responded so effectively to MI-77301 that an additional benefit from combination therapy could not be detected. Our investigations suggest a mechanism of action underlying the combination effect in our models and most probably in prior models. NVP-CGM097 synergised with fulvestrant and the major effect of combination was enhanced downregulation of cell cycle-related transcriptional programmes, particularly the E2F-regulated transcriptome that when unimpeded is activated as cells progress out of $\mathrm{G}_{1}$. Our data suggest that combining MDM2 inhibition with endocrine therapy leads to a profound arrest of cell cycle processes and our in vitro analysis of the MCF-7 FasR cell line supports this finding, consistent with an increase in senescence as a possible mechanism.

CDK4/6 inhibitors in combination with endocrine therapy are the standard of care for advanced breast cancer in the first- and second-line treatment settings. However, resistance to combination therapy will inevitably develop [4], and it is imperative that therapies that can operate in the post resistance setting are identified. We show for the first time that activation of $\mathrm{p} 53^{\mathrm{wt}}$ is effective in a model of CDK4/6 inhibitor-resistant breast cancer. Interestingly, we have demonstrated that p53- induced cell cycle inhibition in palbociclib-resistant MCF-7 is predominantly in the $G_{2}$ phase. CDK4/6 inhibition acts to prevent $G_{1}$ cell cycle entry by preventing the phosphorylation of $\mathrm{Rb}$ [40-42], and many mechanisms of CDK4/6 inhibitor resistance are via disruption of the $G_{1} / S$ checkpoint [4]. Our findings therefore indicate that palbociclib-resistant cell lines that have evaded $G_{1}$ checkpoints are still susceptible to $G_{2}$ arrest. The combination of MDM2 inhibition and CDK4/6 inhibition engage pathways that remain intact in palbociclib and endocrine-resistant cells, unlike the $G_{1} / S$ checkpoint which is frequently disabled in these resistance contexts.

We also show that rechallenging palbociclib-resistant cells with palbociclib in combination with MDM2 inhibition increases B-galactosidase staining and p21 expression, both indicative of senescence. Likewise, fulvestrantresistant cells treated with combination MDM2 inhibition and fulvestrant become senescent. Senescence entry occurs from either $G_{1}$ phase or $G_{2} / M$ phase [43]. Thus, in our models of resistance to either endocrine therapy or palbociclib, NVP-CGM097-mediated induction of $\mathrm{G}_{2} / \mathrm{M}$ accumulation along with the downregulation of FOXM1 and other senescence-related transcripts appears to be sufficient to trigger entry into senescence. CDK4/6 inhibitors are known to increase senescence entry and MDM2 can counteract this [44].

\section{Conclusions}

Collectively, our results suggest that although ER has been shown to be a negative regulator of p53 [14, 45, 46], it is the anti-proliferative actions of fulvestrant (particularly where they overlap with CDK4/6 inhibition) and MDM2 inhibition interacting via cell cycle progression pathways that combine to produce an enhanced anti-tumour effect. Our data provide a preclinical rationale for the combination of MDM2 inhibition with either endocrine therapy or CDK4/6 inhibition, even in the resistant setting. Importantly, we also offer a novel mechanistic insight that will inform the future design of rational combinations with MDM2 inhibitors in ERpositive breast cancer, demonstrating the efficacy of cotargeting cell cycle pathways and perhaps suggesting an alternative strategy via designing combinations to promote the pro-apoptotic functions of p53.

\section{Supplementary information}

Supplementary information accompanies this paper at https://doi.org/10. 1186/s13058-020-01318-2.

Additional file 1: Table S1. Differential expression analysis of MCF7 cell lines following 48 hours treatment with NVP-CGM097 versus vehicle (0.01\% DMSO). Table S2: Differential expression analysis of MCF7 cell lines following 48 hours treatment with Fulvestrant versus vehicle $(0.01 \%$ DMSO). Table S3. Differential expression analysis of MCF7 cell lines following 48 hours treatment with combination therapy (NVP-CGM097 + 
fulvestrant) versus vehicle (0.01\% DMSO). Table S4. Differential genes induced by combination therapy (NVP-CGM097 + fulvestrant) versus vehicle (0.01\% DMSO). Table S5. KEGG PATHWAY enrichment analysis of differentially expressed genes following treatment with combination of NVP-CGM097 and fulvestrant versus vehicle (0.01\% DMSO). Table S6. Combination effect quantified for all genes following treatment with NVP-CGM097, fulvestrant and combination across cell lines. Table S7. Differential expression analysis of the PDX breast cancer model treated with NVP-CGM097 versus vehicle. Table S8. Differential expression analysis of the PDX breast cancer model treated with fulvestrant versus vehicle. Table S9. Differential expression analysis of the PDX breast cancer model treated with combination therapy versus vehicle. Table S10. Gene set enrichment analysis of DE genes induced by NVP-CGM097 in MCF-7 cell lines. Table S11. Gene set enrichment analysis of DE genes induced by fulvestrant in MCF-7 cell lines. Table S12. Gene set enrichment analysis of DE genes induced by combination therapy in MCF-7 cell lines. Table S13. Gene set enrichment analysis of DE genes induced by NVP-CGM097 in the PDX treated model. Table S14. Gene set enrichment analysis of DE genes induced by fulvestrant in the PDX treated model. Table S15. Gene set enrichment analysis of DE genes induced by combination therapy in the PDX treated model. Table S16. Combination effect quantified for all genes following treatment with NVP-CGM097, fulvestrant and combination in the PDX model. Table S17. Differential expression analysis of MCF7 cell lines following 48 hours treatment with NVP-CGM097 versus vehicle (0.01\% DMSO). Table S18. Differential expression analysis of MCF7 cell lines following 48 hours treatment with palbociclib versus vehicle (0.01\% DMSO). Table S19. Differential expression analysis of MCF7 cell lines following 48 hours treatment with combination therapy (NVP-CGM097 + palbociclib) versus vehicle $(0.01 \%$ DMSO). Table S20. Combination effect quantified for all genes following treatment with NVP-CGM097, palbociclib and combination across cell lines.

Additional file 2: Fig. S1 MDM2 inhibition activates p53 and reduces tumour proliferation in vitro and in vivo. A. Full gel and blot scans for the Western blots shown in Fig. 1b. Total protein was visualised using BioRad stain-free imaging technology according to the manufacturer's instructions. B. Analysis of cell cycle phase using flow cytometry to quantify propidium iodide staining of genomic DNA shows significant alterations to cell cycle phase distribution in $\mathrm{p} 53^{\mathrm{wt}}$ models consistent with arrest in both $G_{1}$ and $G_{2}$ after incubation for 48 hours with $1 \mu M$ NVP-CGM097. Red $=G_{1}$ (bottom), blue $=S$ (middle), green $=G_{2} / M$ (top). Statistical significance from $x^{2}$ test using the vehicle treated profile as the expected value is indicated. C. NVP-CGM097 $(50 \mathrm{mg} / \mathrm{kg}$ daily, red) significantly inhibited tumour volumes compared to vehicle (2\% DMSO daily, green) at endpoint. Final tumour volumes were compared using two-tailed T test to determine significance. D. Representative images of Ki-67 quantification of endpoint tumours in Qupath software showing the classification of different tissue compartments: tumour (red and blue), stroma (green), and necrosis (black); and detection of Ki-67 negative and positive tumour cells. A single classifier was applied to all tumour sections. Fig. S2. NVPCGM097 treatment causes gene expression changes in cell cycle and p53 pathways in vitro. A. Multidimensional scaling (MDS) plot showing the level of sample similarity between MCF-7 cell lines treated with vehicle, NVP-CGM097, fulvestrant and combination therapy (NVP-CGM097 plus fulvestrant). B. Venn diagram showing the overlap between differentially expressed genes (adjusted $p$-value $<0.05$ - and 2 -fold change) induced by treatment in MCF-7 cell lines. C. KEGG pathway analysis using RNA-Seq transcriptomic data shows a significant negative enrichment of Cell Cycle regulation in MCF-7 cell lines following 48 hours of treatment with $1 \mu \mathrm{M}$ NVP-CGM097 and positive enrichment of p53 Signalling Pathway. D. KEGG enrichment analysis showing p53 Signalling Pathway activation in MCF-7 cell lines following treatment with NVP-CGM097. Fig. S3 Enrichment analysis of significant differentially expressed genes against curated gene sets. Fig. S4 MDM2 inhibition induces changes in gene and protein expression associated with the cell cycle in endocrine resistant models in vitro and in vivo. A. Incucyte analysis of cell numbers of parental MCF7 cells (blue) and MCF-7 FasR cells (red) over time after treatment with $100 \mathrm{nM}$ fulvestrant. Vehicle treated parental MCF-7 cell growth (grey) is shown for comparison. B. Full gel images for the Western blots shown in Fig. 4c. C. Quantification of ER staining in the nucleus and cytoplasm of three independent replicates per arm from PDX model Gar15-13 following 10 days of treatment with vehicle (2\% DMSO daily, green), NVPCGM097 (50mg/kg daily, red), fulvestrant (5 mg weekly, blue) or the combination of fulvestrant and NVP-CGM097 (purple). Tumours treated with fulvestrant alone or in combination had significantly reduced ER staining in both cellular compartments compared to tumours not treated with fulvestrant. Tumour cell identification and ER quantification were performed in QuPath software. D. Heat map of significant downregulated genes from the E2F Targets and $\mathrm{G}_{2} / \mathrm{M}$ Checkpoint gene sets in the Gar1513 PDX model. E. Normalised $\log _{2}$ expression levels of significant p53 target genes in in the Gar1513 PDX model induced by NVP-CGM097 (red), fulvestrant (blue) and the combination of NVP-CGM097 and fulvestrant (purple), averaged by condition, associated with cell cycle, apoptosis and senescence. Fig. S5. MDM2 inhibition in combination with CDK4/6 inhibition synergistically downregulates cell cycle markers in vitro. A. MDS plot of MCF-7 cell lines treated for $48 \mathrm{~h}$ with Vehicle (0.01\% DMSO), $1 \mu \mathrm{M}$ NVP-CGM097, $500 \mathrm{nM}$ palbociclib, or the combination of NVP-CGM097 and palbociclib. B. Venn diagram showing the overlap between differentially expressed genes (adjusted $p$-value $<0.05$ - and 2 -fold change) induced by treatment in MCF-7 cell lines. C. Viability of parental MCF-7 (grey) and MCF-7 PalbR (orange) after treatment with the indicated dose of palbociclib for $72 \mathrm{~h}$. Values are shown relative to vehicle treated cells. D. Full gel images for the Western blots shown in Fig. $7 \mathrm{~d}$.

\section{Abbreviations}

ER: Oestrogen Receptor; CDK4/6: Cyclin-dependent kinase 4 and 6; C.I: Combination index; BrdU: Bromodeoxyuridine; P.I: Propidium iodide; IHC: Immunohistochemistry; GSEA: Gene set enrichment analysis; GO: Gene ontology; MSigDB: Molecular Signatures Database; KEGG: Kyoto Encyclopedia of Genes and Genomes; (T)p53wt: p53 (or TP53) wildtype; (T)p53mut: p53 (or TP53) mutant; IC50: Half maximal inhibitory concentration; RNASeq: RNA sequencing; PDX: Patient-derived xenograft; FasR: Fulvestrant (Faslodex) resistant; PalbR: Palbociclib resistant

\section{Acknowledgements}

Not applicable

\section{Authors' contributions}

NP and HHM designed and performed experiments, analysed the results and wrote the manuscript. EL and CEC analysed the results and wrote the manuscript. SA, RC, AY and KJF designed and performed the experiments and analysed the results. KMC, AS, SH and YH analysed the results and provided critical feedback on the manuscript. AP provided critical reagents, oversight of $\mathrm{HC}$ analyses and critical feedback on the manuscript, DS provided critical reagents and EH and WDT provided intellectual input during the conception of the project and critical feedback on the manuscript. The authors read and approved the final manuscript.

\section{Funding}

Novartis Australia (NP, HM, EL); National Breast Cancer Foundation (NP: IIRS19-053, EL: EC17-002 and PRAC14-02); Cancer Institute of New South Wales (HM: ECF171156); Breast Cancer Trials - Australia and New Zealand (EL); The Estate of the late RT Hall (AS); Love Your Sister (EL).

\section{Availability of data and materials}

The datasets supporting the conclusions of this article are included within the article and its additional files. The read count quantitation and normalised expression matrix for all RNAseq experiments can be downloaded from http://www.ncbi.nlm.nih.gov/geo (GSE140758). Cell lines and PDX models not commercially available can be obtained via MTA with Garvan Institute of Medical Research.

\section{Ethics approval and consent to participate}

All in vivo experiments, procedures and endpoints were approved by the Garvan Institute of Medical Research Animal Ethics Committee (protocol 15/ 25). PDX models were collected with patient consent under approval from Royal Prince Alfred Hospital human research ethics committee (HREC/13/ RPA/187 - KCC P 3837) and St Vincent's Hospital human research ethics committee (HREC/H2006/2633 - Gar15-13). 


\section{Consent for publication}

Not applicable.

\section{Competing interests}

Funding and drugs for this study were provided under a research agreement with Novartis Australia.

EL provides advisory board services to Novartis Australia, Roche Australia, Specialised Therapeutics Australia, Pfizer Australia, Lilly Australia and Amgen Australia (All honoraria paid to the Garvan Institute of Medical Research). $\mathrm{EH}$ is an employee at Novartis.

\section{Author details}

${ }^{1}$ Garvan Institute of Medical Research, Darlinghurst, Sydney, NSW 2010, Australia. ${ }^{2}$ St. Vincent's Clinical School, Faculty of Medicine, University of New South Wales Sydney, Sydney, NSW 2010, Australia. ${ }^{3}$ Tumor Suppression Laboratory, Peter MacCallum Cancer Centre, 305 Grattan St, Melbourne, VIC 3000, Australia. ${ }^{4}$ Sir Peter MacCallum Department of Oncology, The University of Melbourne, Parkville, VIC 3010, Australia. ${ }^{5}$ Novartis Institutes of Biomedical Research, Cambridge, MA, USA. ${ }^{6}$ Adelaide Medical School, The University of Adelaide, Adelaide, SA 5001, Australia.

Received: 5 February 2020 Accepted: 21 July 2020

Published online: 12 August 2020

\section{References}

1. Lim E, Tarulli G, Portman N, Hickey TE, Tilley WD, Palmieri C. Pushing estrogen receptor around in breast cancer. Endocr Relat Cancer. 2016; 23(12):T227-T41.

2. Hayes EL, Lewis-Wambi JS. Mechanisms of endocrine resistance in breast cancer: an overview of the proposed roles of noncoding RNA. Breast Cancer Res. 2015;17:40.

3. Turner NC, Slamon DJ, Ro J, Bondarenko I, Im S-A, Masuda N, et al. Overall survival with palbociclib and fulvestrant in advanced breast cancer. $\mathrm{N}$ Engl $J$ Med. 2018;379(20):1926-36

4. Portman, N., Alexandrou, S., Carson, E., Wang, S., Lim, E., Caldon, C.E. Overcoming CDK4/6 inhibitor resistance in ER-positive breast cancer. Endocrine-related cancer. 2019;26(1):R15-R30.

5. Burgess A, Chia KM, Haupt S, Thomas D, Haupt Y, Lim E. Clinical overview of MDM2/X-targeted therapies. Front Oncol. 2016;6:7.

6. Haupt S, Vijayakumaran R, Miranda PJ, Burgess A, Lim E, Haupt Y. The role of MDM2 and MDM4 in breast cancer development and prevention. J Mol Cell Biol. 2017;9(1):53-61.

7. Hock AK, Vousden $\mathrm{KH}$. The role of ubiquitin modification in the regulation of p53. Biochim Biophys Acta. 2014;1843(1):137-49.

8. Haupt Y, Maya R, Kazaz A, Oren M. Mdm2 promotes the rapid degradation of p53. Nature. 1997;387(6630):296-9.

9. Kubbutat $\mathrm{MH}$, Jones $\mathrm{SN}$, Vousden $\mathrm{KH}$. Regulation of p53 stability by Mdm2. Nature. 1997:387(6630):299-303.

10. Li M, Brooks CL, Wu-Baer F, Chen D, Baer R, Gu W. Mono- versus polyubiquitination: differential control of p53 fate by Mdm2. Science. 2003; 302(5652):1972-5.

11. Yu Q, Li Y, Mu K, Li Z, Meng Q, Wu X, et al. Amplification of Mdmx and overexpression of MDM2 contribute to mammary carcinogenesis by substituting for p53 mutations. Diagn Pathol. 2014;9:71.

12. Swetzig WM, Wang J, Das GM. Estrogen receptor alpha (ERa/ESR1) mediates the p53-independent overexpression of MDM4/MDMX and MDM2 in human breast cancer. Oncotarget. 2016;7(13):16049.

13. Dolfi SC, Jäger AV, Medina DJ, Haffty BG, Yang J-M, Hirshfield KM. Fulvestrant treatment alters MDM2 protein turnover and sensitivity of human breast carcinoma cells to chemotherapeutic drugs. Cancer Lett. 2014;350(1-2):52-60.

14. Bailey ST, Shin H, Westerling T, Liu XS, Brown M. Estrogen receptor prevents p53-dependent apoptosis in breast cancer. Proc Natl Acad Sci. 2012;109(44): 18060-5.

15. Caldon CE. Estrogen signaling and the DNA damage response in hormone dependent breast cancers. Front Oncol. 2014;4:106

16. Lu J, McEachern D, Li S, Ellis MJ, Wang S. Reactivation of p53 by mdm2 inhibitor mi-77301 for the treatment of endocrine-resistant breast cancer. Mol Cancer Ther. 2016;15(12):2887-93.

17. Holzer P, Masuya K, Furet P, Kallen J, Valat-Stachyra T, Ferretti Sp, et al. Discovery of a dihydroisoquinolinone derivative (NVP-CGM097): a highly potent and selective MDM2 inhibitor undergoing phase 1 clinical trials in p53wt tumors. J Med Chem. 2015;58(16):6348-58.

18. Bauer S, Demetri G, Jeay S, Dummer R, Guerreiro N, Tan DS, et al. 366PD - A phase I, open-label, multi-center, dose escalation study of oral NVP-CGM097, a p53/HDM2-protein-protein interaction inhibitor, in adult patients with selected advanced solid tumors. Ann Oncol. 2016:27(S6):vi116.

19. Chou TC, Talalay P. Quantitative analysis of dose-effect relationships: the combined effects of multiple drugs or enzyme inhibitors. Adv Enzym Regul. 1984;22:27-55.

20. Chia K, Milioli H, Portman N, Laven-Law G, Coulson R, Yong A, et al. Noncanonical AR activity facilitates endocrine resistance in breast cancer. Endocr Relat Cancer. 2019;26(2):251-64.

21. Oakes SR, Vaillant F, Lim E, Lee L, Breslin K, Feleppa F, et al. Sensitization of $\mathrm{BCL}-2$-expressing breast tumors to chemotherapy by the $\mathrm{BH} 3$ mimetic $\mathrm{ABT}$ 737. Proc Natl Acad Sci. 2012;109(8):2766-71.

22. Liao Y, Smyth GK, Shi W. featureCounts: an efficient general purpose program for assigning sequence reads to genomic features. Bioinformatics. 2013;30(7):923-30

23. Robinson MD, McCarthy DJ, Smyth GK. edgeR: a bioconductor package for differential expression analysis of digital gene expression data. Bioinformatics. 2010:26(1):139-40.

24. McCarthy DJ, Chen Y, Smyth GK. Differential expression analysis of multifactor RNA-Seq experiments with respect to biological variation. Nucleic Acids Res. 2012;40(10):4288-97.

25. Ritchie ME, Phipson B, Wu D, Hu Y, Law CW, Shi W, et al. limma powers differential expression analyses for RNA-sequencing and microarray studies. Nucl Acids Res. 2015;43(7):e47-e.

26. Yu G, Wang L-G, Han Y, He Q-Y. clusterProfiler: an R package for comparing biological themes among gene clusters. Omics. 2012;16(5):284-7.

27. Reuther C, Heinzle V, Nolting S, Herterich S, Hahner S, Halilovic E, et al. The HDM2 (MDM2) inhibitor NVP-CGM097 inhibits tumor cell proliferation and shows additive effects with 5-fluorouracil on the p53-p21-Rb-E2F1 cascade in the p53wild type neuroendocrine tumor cell line GOT1. Neuroendocrinology. 2018;106(1):1-19.

28. Weisberg E, Halilovic E, Cooke VG, Nonami A, Ren T, Sanda T, et al. Inhibition of wild-type p53-expressing AML by the novel small molecule HDM2 inhibitor CGM097. Mol Cancer Ther. 2015;14(10): 2249-59.

29. Holzer P, Masuya K, Furet P, Kallen J, Valat-Stachyra T, Ferretti S, et al. Discovery of a dihydroisoquinolinone derivative (NVP-CGM097): a highly potent and selective MDM2 inhibitor undergoing phase 1 clinical trials in p53wt tumors. J Med Chem. 2015;58(16):6348-58.

30. Tao YF, Wang NN, Xu LX, Li ZH, Li XL, Xu YY, et al. Molecular mechanism of G1 arrest and cellular senescence induced by LEE011, a novel CDK4/CDK6 inhibitor, in leukemia cells. Cancer Cell Int. 2017;17:35.

31. Xie Q, Wu Q, Mack SC, Yang K, Kim L, Hubert CG, et al. CDC20 maintains tumor initiating cells. Oncotarget. 2015:6(15):13241-54.

32. Laoukili J, Kooistra MR, Bras A, Kauw J, Kerkhoven RM, Morrison A, et al. FoxM1 is required for execution of the mitotic programme and chromosome stability. Nat Cell Biol. 2005;7(2):126-36.

33. Vilgelm AE, Saleh N, Shattuck-Brandt R, Riemenschneider K, Slesur L, Chen SC, et al. MDM2 antagonists overcome intrinsic resistance to CDK4/6 inhibition by inducing p21. Sci Transl Med. 2019;11(505):eaav7171.

34. Patnaik A, Rosen LS, Tolaney SM, Tolcher AW, Goldman JW, Gandhi L, et al. Efficacy and safety of Abemaciclib, an inhibitor of CDK4 and CDK6, for patients with breast cancer, non-small cell lung cancer, and other solid tumors. Cancer Discov. 2016;6(7):740-53.

35. Tisato V, Voltan R, Gonelli A, Secchiero P, Zauli G. MDM2/X inhibitors under clinical evaluation: perspectives for the management of hematological malignancies and pediatric cancer. J Hematol Oncol. 2017;10(1):133.

36. Nair $A B$, Jacob $S$. A simple practice guide for dose conversion between animals and human. J Basic Clin Pharm. 2016:7(2):27-31.

37. Spring LM, Wander SA, Andre F, Moy B, Turner NC, Bardia A. Cyclindependent kinase 4 and 6 inhibitors for hormone receptor-positive breast cancer: past, present, and future. Lancet. 2020;395(10226):817-27.

38. Jeay S, Ferretti S, Holzer P, Fuchs J, Chapeau EA, Wartmann M, et al. Dose and schedule determine distinct molecular mechanisms underlying the efficacy of the p53-MDM2 inhibitor HDM201. Cancer Res. 2018;78(21):6257-67.

39. Fischer M, Quaas M, Steiner L, Engeland K. The p53-p21-DREAM-CDE/ CHR pathway regulates G2/M cell cycle genes. Nucleic Acids Res. 2016; 44(1):164-74 
40. Petrossian K, Kanaya N, Lo C, Hsu PY, Nguyen D, Yang L, et al. ERalphamediated cell cycle progression is an important requisite for CDK4/6 inhibitor response in HR+ breast cancer. Oncotarget. 2018;9(45):27736-51.

41. Prall OW, Rogan EM, Sutherland RL. Estrogen regulation of cell cycle progression in breast cancer cells. J Steroid Biochem Mol Biol. 1998;65(1-6): 169-74.

42. Foster JS, Wimalasena J. Estrogen regulates activity of cyclin-dependent kinases and retinoblastoma protein phosphorylation in breast cancer cells. Mol Endocrinol. 1996;10(5):488-98.

43. Sharpless NE, Sherr CJ. Forging a signature of in vivo senescence. Nat Rev Cancer. 2015;15(7):397-408.

44. Klein ME, Dickson MA, Antonescu C, Qin LX, Dooley SJ, Barlas A, et al. PDLI $\mathrm{M} 7$ and $\mathrm{CDH} 18$ regulate the turnover of MDM2 during CDK4/6 inhibitor therapy-induced senescence. Oncogene. 2018;37(37):5066-78.

45. Konduri SD, Medisetty R, Liu W, Kaipparettu BA, Srivastava P, Brauch H, et al. Mechanisms of estrogen receptor antagonism toward p53 and its implications in breast cancer therapeutic response and stem cell regulation. Proc Natl Acad Sci U S A. 2010;107(34):15081-6.

46. Sayeed A, Konduri SD, Liu W, Bansal S, Li F, Das GM. Estrogen receptor alpha inhibits p53-mediated transcriptional repression: implications for the regulation of apoptosis. Cancer Res. 2007;67(16):7746-55.

\section{Publisher's Note}

Springer Nature remains neutral with regard to jurisdictional claims in published maps and institutional affiliations.

Ready to submit your research? Choose BMC and benefit from:

- fast, convenient online submission

- thorough peer review by experienced researchers in your field

- rapid publication on acceptance

- support for research data, including large and complex data types

- gold Open Access which fosters wider collaboration and increased citations

- maximum visibility for your research: over $100 \mathrm{M}$ website views per year

At $\mathrm{BMC}$, research is always in progress.

Learn more biomedcentral.com/submissions 